\title{
Unemployment Insurance Fraud and Optimal Monitoring*
}

\author{
David L. Fuller $†$ B. Ravikumar; and Yuzhe Zhang§
}

February 2014

\begin{abstract}
We present evidence that fraudulent collection of unemployment benefits by workers who are gainfully employed is the most relevant incentive problem for the design of unemployment insurance. We show how to efficiently use a combination of tax/subsidy and monitoring to prevent such fraud. The optimal policy monitors the unemployed at fixed intervals. Employment tax is nonmonotonic: it increases between verifications but decreases after a verification. Unemployment benefits are relatively flat between verifications but decrease sharply after a verification. Our quantitative analysis indicates that the optimal auditing cost is less than $60 \%$ of the cost in the current U.S. system. Using the same resources as the U.S. system, the optimal contract delivers a consumption gain of $1.55 \%$ for the average worker.
\end{abstract}

JEL Classification Numbers: D82, D86, J65.

Keywords and Phrases: Unemployment Insurance, Fraud, Concealed Earnings, Costly State Verification.

${ }^{*}$ We are grateful to Árpád Ábrahám, Nicola Pavoni, seminar participants at the Federal Reserve Bank of St. Louis, University of Missouri, and Toulouse School of Economics, and participants at the Workshop on Macroeconomic Applications of Dynamic Games and Contracts, Midwest Macroeconomics Meeting, Midwest Theory Meeting, Asia Meeting of the Econometric Society, and Society for the Advancement of Economic Theory Conference for their helpful comments. We would also like to thank George Fortier for editorial assistance. The views expressed in this article are those of the authors and do not necessarily reflect the views of the Federal Reserve Bank of St. Louis or the Federal Reserve System.

${ }^{\dagger}$ Department of Economics, Concordia University, and CIREQ. Email: david.fuller@concordia.ca

${ }^{\ddagger}$ Research Division, Federal Reserve Bank of St. Louis. Email: b.ravikumar@wustl.edu

$\S$ Department of Economics, Texas A\&M University. Email: yuzhe-zhang@econmail.tamu.edu 


\section{Introduction}

Unemployment insurance programs insure workers against the risk that they may lose their job through no fault of their own. Such insurance, however, is associated with many potential incentive problems. One such incentive problem is moral hazard. For instance, in the presence of insurance, unemployed agents might reduce the search effort, thereby reducing the speed of transition to gainful employment. The literature on the optimal provision of unemployment insurance (e.g., Baily (1978), Shavell and Weiss (1979), and Hopenhayn and Nicolini (1997)) concentrates on providing incentives for optimal search effort. In this paper, we study the incentive problem associated with fraudulent collection of unemployment benefits.

There are several types of unemployment insurance fraud. Examples include collecting unemployment benefits after quitting a job, while being employed, or after refusing a suitable offer. Table 1 below illustrates the overpayments incurred by the unemployment insurance program for different types of fraud.

Table 1: Unemployment Insurance Overpayments in the U.S., 2007

\begin{tabular}{l|c}
\hline Cause & Percent of Fraud Overpayments \\
\hline Concealed Earnings & 60.06 \\
Insufficient Job Search & 4.95 \\
Refused Suitable Offer & 0.80 \\
Quits & 7.06 \\
Fired & 13.29 \\
Unavailable for Work & 4.17 \\
Other & 9.67 \\
Total & 100.00 \\
\hline \hline
\end{tabular}

Source: Benefit Accuracy Measurement program, U.S. Department of Labor

Our focus here is on concealed earnings: the case where an agent currently collecting benefits finds a job, but continues collecting unemployment benefits. As noted in the Table, the benefits overpaid because of such fraud, in 2007, were ten times the overpayment due 
to unemployed agents not actively searching or refusing suitable work. (See Appendix A for more details.) That is, even if the unemployment insurance program were designed to elicit sufficient effort by each unemployed agent, the savings for the program would be dwarfed by the corresponding savings generated by an unemployment insurance program designed to prevent fraud. Furthermore, Ashenfelter, Ashmore, and Deschenes (2005) use randomized trials in four U.S. sites and find that insufficient job search is not a significant source of unemployment insurance overpayments.

The contribution of our paper is to provide an optimal unemployment insurance mechanism in an environment where an agent can commit fraud by concealing his true employment status. All of the agents in our model have constant absolute risk aversion (CARA) preferences, are initially unemployed, and face stochastic opportunities to transition to employment. Those who are employed receive positive wage income, while those who remain unemployed receive zero wage income. There is no search effort to find employment. Employment is assumed to be an absorbing state. The employed agent could conceal the fact that he has found a job and continue to claim unemployment benefits. The Unemployment Insurance agency has a costly monitoring technology to detect the agent's employment status. Our mechanism efficiently provides benefits and utilizes the monitoring technology to deter potential fraud.

We set up the problem in continuous time and represent incentive constraints as differential equations, similar to Zhang (2009). We then formulate the contracting problem as one of optimal control and apply the Pontryagin minimum principle to study the dynamics of unemployment insurance policies. We deliver a pre-commitment mechanism that optimally provides unemployment insurance in the presence of persistent private information.

In our model, the Unemployment Insurance agency uses two instruments to deter fraud: tax/subsidy and monitoring. Both instruments are costly. The first instrument distorts consumption relative to full insurance. The second instrument has a direct cost.

Since employment is an absorbing state in our model, the treatment of the worker who transitioned to employment is straightforward - constant consumption forever and no monitoring. Since employment status is private information, the Unemployment Insurance agency distorts consumption and does not fully insure the unemployed worker. The 
Unemployment Insurance agency also uses the monitoring instrument to provide incentives.

We consider two monitoring mechanisms: deterministic verification and stochastic verification. Under deterministic verification, the worker is either verified with probability one or not verified at all. We focus on this case for most of the paper since it is analytically tractable. All of our results below are for this case. We show later that our results remain the same under stochastic verification where the worker is verified with probability between zero and one. That is, even though our deterministic mechanism appears restrictive, the general mechanism of stochastic verification does not offer more economic insights on unemployment insurance and monitoring.

We show that under deterministic verification the interval between consecutive monitoring periods is a constant, independent of history. That is, the monitoring consists of cycles and occurs in pre-specified periods. With CARA preferences, the worker's utility flows in a new cycle are proportional to those in the previous cycle. Hence, his incentive to commit fraud remains the same and he is monitored in the same manner as in the previous cycle.

The optimal unemployment benefits in our model decrease monotonically with the duration of unemployment. Benefits remain relatively flat between verifications with a sharp decline immediately after a verification.

A novel feature of our optimal mechanism is that it implies a nonmonotonic tax on employment in order to provide incentives. Within a monitoring cycle, the consumption for the worker who transitions to employment earlier exceeds that of the worker who transitions later. However, the consumption for the worker who transitions to employment shortly before a verification period is less than that of the worker who transitions shortly after.

Another type of fraud is that employed agents can quit their jobs, become unemployed, and start collecting unemployment benefits. Even though an employed agent in our model can commit such fraud, the incentives in our optimal contract ensure that the agent does not engage in such fraudulent behavior.

We perform a quantitative analysis similar to Hopenhayn and Nicolini (1997) and find that the optimal auditing cost is less than 60 percent of the cost incurred by the U.S. unemployment insurance system. Furthermore, using the same resources as the U.S. system the 
optimal contract delivers higher utility to the average worker: 1.55 percent higher consumption at every date. This gain arises from two sources: (i) improved consumption smoothing between employed and unemployed states and (ii) reduced monitoring costs or higher average consumption. The U.S. system spends only 0.24 percent its resources on monitoring the average worker and spends the rest on unemployment benefits (net of wages), but the same resources are allocated differently in the optimal contract: 0.17 percent is spent on monitoring the average worker and the rest is spent on unemployment benefits. Thus, almost all of the gain in the optimal contract comes from improved consumption smoothing.

Several remarks are in order here regarding our model choices and concealed earnings fraud. First, preventing concealed earnings fraud in our model is a matter of inducing an unemployed worker who finds a job not to delay reporting the transition to employment. One might think such fraud could be automatically detected by cross-matching the unemployment insurance records with the employment records. Indeed, the cross-matching technology has been available to the unemployment insurance agencies in every state for more than a decade. (Recall that unemployment insurance is administered at the state level in the U.S. and each state has its own eligibility rules.) However, as noted in Table A.2, Appendix A, only 7.5 percent of the fraud cases are detected by cross-matching with the state's directory of new hires. For instance, cross-matching technology would not automatically catch a worker who is collecting unemployment benefits in one state, but is employed in another state. Furthermore, even within each state, since the directory of new hires is updated monthly, some workers who truthfully report unemployment in a specific week may show up in a cross-match of employment records and be mistakenly flagged for fraud. In most cases when a worker appears in both unemployment insurance records and employment records, further investigation is necessary to determine if fraud has actually occurred.

Second, the worker could commit a more nuanced form of concealed earnings fraud by truthfully reporting the transition to employment but underreporting the earnings. (The worker could collect some unemployment benefits as long as the reported earnings are sufficiently low.) In 2007, according to the Benefit Accuracy Measurement (BAM) program data, roughly 40 percent of those committing concealed earnings fraud reported positive 
earnings. Again, an automatic technology that cross-matches the unemployment insurance records with wage records (updated quarterly) in each state would not be sufficient to detect most of the cases of concealed earnings fraud; In 2007, less than 2 percent of the cases were caught using such a cross-match (see Table A.2, Appendix A). In fact, employees working in a sector not covered by the UI system will never show up in the State Wage records (e.g. federal employees, self-employed). Clearly, more than 90 percent of concealed earnings fraud was missed by the cross-matching technology that uses both the directory of new hires and the wage records. The detection involves a case-by-case investigation and, thus, a per-case cost as in our model, and our mechanism offers an optimal approach to deter the fraud. ${ }^{1}$

Third, a form of concealed earnings fraud could be collecting unemployment benefits while working "off-the-books" where the worker is paid in cash. The BAM data, however, suggests that the fraud is occurring when the worker is in "official" or "on-the-books" employment. While the worker is committing concealed earnings fraud, his weekly earnings are similar to the weekly earnings in the pre-unemployment job (which, by design, has to be official for the worker to collect unemployment benefits). In 2007, for example, those committing the fraud were earning 82 percent of their previous job's wages, on average. One-fourth of those committing the fraud were earning more while collecting benefits than before they became unemployed. Earnings of this magnitude are suggestive of official employment. $^{2}$

Fourth, we do not have search effort in our model, so we do not provide incentives for finding employment. One could argue that providing incentives for the search effort could result in more cost savings, relative to fraud detection, by moving agents from the unemployed pool to the employed pool at a faster rate. However, this margin turns out to

\footnotetext{
${ }^{1}$ Our model does not allow for a strategic choice of the earnings to report, but can nevertheless be used to examine the nuanced fraud. The incentives to cheat in our model depend on the difference in wages between two states: employed and unemployed. The latter is normalized to zero in our analysis, but we can easily replicate the analysis for a positive wage in the unemployed state and for a different wage gap between the two states.

${ }^{2}$ There is an interview component to the BAM program that may capture some cash earnings, but these cases are at most 10.5 percent of the sample (see Table A.2, Appendix A).
} 
be quantitatively insignificant in the previous models of optimal unemployment insurance. In Hopenhayn and Nicolini (1997), for instance, the unemployed agent's optimal search effort and the optimal duration of unemployment are almost the same as those in the current unemployment insurance program; in Wang and Williamson (2002), the optimal insurance does not encourage more job search effort than what is implied by the current unemployment insurance program. The cost savings in these models come from reduced benefits on average and better distribution of benefits based on duration of unemployment. This channel of cost savings is present in our model as well.

Finally, a strand of the unemployment insurance literature has focused on monitoring search effort and/or monitoring acceptance of job offers. Hansen and Imrohoroglu (1992), for instance, examine a model where agents can reject job offers. An exogenous fraction of agents who reject job offers are denied benefits. In our optimal mechanism, the unemployed agent who receives an opportunity to become employed has no incentive to refuse the opportunity. Setty (2011) analyzes an optimal unemployment insurance scheme in a model where the agent's search effort is monitored. In our model, we use the monitoring technology to detect concealed earnings. Empirically, as noted in Table 1, fraudulent behavior in search effort or refusal of suitable work is not as costly to the Unemployment Insurance agency as concealment of earnings.

The remainder of the paper proceeds as follows. In Section 2 we describe the model. In Section 3 we establish two properties of the optimal mechanism: scaling and periodic monitoring. In Section 4 we use these properties to analyze the optimal unemployment insurance scheme with exogenously given monitoring dates. Then, we characterize the optimal monitoring dates in Section 5. In Section 6 we show that our mechanism prevents employed workers from quitting. In Section 7 we discuss an extension of our model to the stochastic monitoring case, where the Unemployment Insurance agency optimally chooses the probability of verification. In this section, we also describe the similarities and differences between the insights from the deterministic mechanism and the insights from the stochastic mechanism. We conclude in Section 8. 


\section{$2 \quad$ Model}

The Unemployment Insurance authority is a risk-neutral principal with a discount rate $r>0$. She provides insurance to a risk-averse worker, whose preferences are given by

$$
E\left[\int_{0}^{\infty} e^{-r t} r v(c(t)) d t\right]
$$

where $c(t)$ is consumption at time $t, v(c)=-e^{-\rho c}$ is a CARA utility function with risk aversion $\rho, r$ is the discount rate, and $E$ is the expectation operator. Note that the flow utility is $r v(c)$ and that the agent's subjective discount rate is the same as the principal's.

A worker can be either employed with wage $w>0$ or unemployed with wage zero. The worker is unemployed at $t=0$ and transitions to employment with Poisson rate $\pi>0$. We assume that employment is permanent. (For similar assumptions, see the unemployment insurance model of Hopenhayn and Nicolini (1997) and the disability insurance model of Golosov and Tsyvinski (2006).)

The worker's employment status is private information, so an employed worker can claim to be unemployed and continue collecting the unemployment benefits. We refer to this as fraud. The principal can verify the worker's unemployment report at a cost of $\gamma$ units of the consumption good. ${ }^{3}$ Verification reveals the worker's true employment status.

We study pre-commitment mechanisms that efficiently deliver unemployment benefits and deter fraud. In addition to the tax/subsidy instrument used by the unemployment insurance literature, our mechanism uses the monitoring instrument to provide incentives.

We assume that the principal always collects the wage, so an unemployed worker can never claim to be employed. Hence, there is no need for verification when the worker reports a transition to employment. Furthermore, since employment is an absorbing state, verification is unnecessary forever if the worker reports to be employed just once in the past. The incentive problem then reduces to ensuring that an employed worker does not claim to be unemployed.

We focus on deterministic verification mechanisms: in each period the worker is either

\footnotetext{
${ }^{3}$ As noted in the Introduction, more than 90 percent of the overpayments due to concealed earnings were not easily detectable by the State authorities using standard procedures available to them. See Appendix A for technologies used to detect Concealed Earnings fraud.
} 
verified with probability one or not verified at all. This mechanism is sub-optimal; it is dominated by a stochastic verification mechanism in our environment. One may then ask why study the deterministic case? Our goal is to characterize the optimal combination of the two instruments: tax/subsidy and monitoring. In Section 7, we show that the key economic insights on these two instruments are nearly identical in both the deterministic and stochastic cases. In both cases, optimal monitoring and employment tax have the same pattern. The stochastic monitoring case requires cumbersome notation and provides less intuition so we start by analyzing the deterministic case.

In our deterministic mechanism, the verification in any period is based on the history of employment status reports and past verifications outcomes. Since verification is necessary only for agents who have been reporting unemployment in every period in the past, a sufficient statistic for past history is the duration of unemployment reports. In other words, at $t=0$ the principal commits to all future verification periods, mapping durations of unemployment reports to $\{0,1\}$. In a verification period, clearly no worker would misreport. (Any penalty $\epsilon>0$ induces truth telling in the verification period.) Thus, the principal does not have to keep track of the outcomes of past verifications. We represent the set of verification periods as $\left\{m_{i} ; i=1,2, \ldots\right\}$, where $m_{i}$ is the date of the $i$ th verification. ${ }^{4}$

The timing is as follows. In the initial period, the worker is unemployed. Then the stochastic job opportunity arrives. The worker either remains unemployed or transitions to employment. He then chooses to report either employment or unemployment to the principal. Conditional on the unemployment report, the principal verifies the true employment status if the period is a verification period. Then, conditional on the report and the outcome of the verification, the principal assigns current and future consumptions. In subsequent periods, if the worker reported employment in the past, he is in an absorbing state and no further reports are necessary. If the worker reported unemployment in every period in the past, then the sequence of events is the same as in the initial period.

If an unemployed worker transitions to employment at $t$, let $c^{E}(t, s)$ denote his con-

\footnotetext{
${ }^{4}$ There is no loss of generality in assuming a countable collection of verification periods. Since each verification costs $\gamma>0$, the principal would not want to verify infinitely many times in any finite time interval.
} 
sumption at time $s \geq t$. Because the principal and the worker have the same discount rate and employment is an absorbing state, efficiency requires that the worker's consumption remain constant after $t$ for all $s$. We therefore suppress $s$ in $c^{E}(t, s)$ and denote this constant level of consumption as $c^{E}(t)$. The flow utility from this level of consumption then is $r v\left(c^{E}(t)\right)$. We denote the discounted sum of utilities to a worker who accepts a job offer for the first time at $t$ as $E(t)$, i.e., $E(t)=\int_{t}^{\infty} e^{-r(s-t)} r v\left(c^{E}(t)\right) d s=v\left(c^{E}(t)\right)$. Since employment status is private information, $E(t)$ is also the continuation utility to a worker who accepted an offer before $t$, but reports employment for the first time at $t$.

An unemployed worker's consumption at $t$ is denoted by $c^{U}(t)$ and his flow utility is $r v\left(c^{U}(t)\right)$. His continuation utility,

$$
U(t) \equiv \int_{t}^{\infty} e^{-r(x-t)} e^{-\pi(x-t)} r v\left(c^{U}(x)\right) d x+\int_{t}^{\infty} e^{-r(x-t)} e^{-\pi(x-t)} \pi E(x) d x,
$$

is the sum of expected utilities before and after the transition $\left(e^{-\pi(x-t)}\right.$ in the first integral is the conditional probability of remaining unemployed at date $x$ and $e^{-\pi(x-t)} \pi$ in the second integral is the density function of the transition time). Hence,

$$
\begin{aligned}
U(t) & =\int_{t}^{\infty} e^{-(r+\pi)(x-t)}(\pi E(x)+r u(x)) d x \\
& =\int_{t}^{s} e^{-(r+\pi)(x-t)}(\pi E(x)+r u(x)) d x+e^{-(r+\pi)(s-t)} U(s), \text { for all } t<s,
\end{aligned}
$$

where $u(x) \equiv v\left(c^{U}(x)\right)$. We will refer to (1) as promise-keeping constraints.

The principal commits at $t=0$ to verification periods $\left\{m_{i} ; i=1,2, \ldots\right\}$ and consumptions $\left\{\left(c^{E}(t), c^{U}(t)\right) ; t \geq 0\right\}$. The verification periods and consumptions are history dependent. We denote this pre-commitment contract as $\sigma$.

Incentive compatibility requires that a worker who transitioned to employment at $t \in$ $\left(m_{i}, m_{i+1}\right)$ does not have the incentive to delay the report of the transition to a later time $s \in\left(t, m_{i+1}\right)$, i.e., report unemployment and commit fraud from $t$ to $s$, and then report employment from $s$ onward:

$$
E(t) \geq \int_{t}^{s} e^{-r(x-t)} r v\left(c^{U}(x)+w\right) d x+e^{-r(s-t)} E(s), \forall s \in\left(t, m_{i+1}\right) .
$$

Note that the worker cannot delay the report beyond the next verification period $m_{i+1}$. 
We restrict contract allocations to

$$
E(t) \geq U(t) \text {, for all } t .
$$

Restriction (3) rules out the fraud due to refusal of offers noted in Table 1 (0.8 percent of total fraud overpayments). This restriction can be derived by adding a job-refusal option to our model. For ease of exposition we have imposed the restriction on the mechanism; Appendix B describes the job-refusal option and derives this restriction.

The expected cost for the principal is

$$
C(\sigma)=\int_{0}^{\infty} e^{-(r+\pi) t}\left(\pi c^{E}(t)+r c^{U}(t)\right) d t+\sum_{i} e^{-(r+\pi) m_{i}} \gamma .
$$

There should, in fact, be an additional term in $C(\sigma)$ : the discounted income obtained by the principal, $\frac{\pi w}{r+\pi}$. However, unlike the unemployment insurance literature that endogenizes job-finding probabilities, the discounted income in our model is a constant, so it does not affect the optimal $\sigma$.

The principal's problem is to find an incentive compatible $\sigma$ that minimizes $C(\sigma)$ and delivers the initial promised utility $U(0)$, i.e.,

$$
\begin{array}{cl}
\min _{\sigma} & C(\sigma) \\
\text { subject to } & U(0)=\int_{0}^{\infty} e^{-(r+\pi) t}(\pi E(t)+r u(t)) d t, \\
& \text { and constraints }(2),(3) .
\end{array}
$$

With a slight abuse of notation, denote the principal's cost function as $C(U(0)) .{ }^{5}$

\section{A Simplification of the Optimal Contract}

We begin our analysis by presenting two features of the optimal contract. In Section 3.1 we establish a "scaling" property. Then, in Section 3.2 we show that the optimal monitoring

\footnotetext{
${ }^{5}$ Ravikumar and Zhang (2012) analyze the problem of tax compliance in a costly state verification model where the verification technology is imperfect (a low-income agent might be mistakenly labeled as high income). They solve for the principal's cost function using the Hamilton-Jacobi-Bellman equation. In contrast, we study optimal unemployment insurance in an environment with a perfect verification technology. We characterize the path of unemployment benefits by formulating the optimal control problem and using the Pontryagin minimum principle.
} 
is periodic. These properties simplify our analysis of the optimal contract by narrowing the search of a solution to problem (4) to a smaller space.

To help us simplify, we rewrite problem (4) in terms of continuation utilities $E(\cdot), U(\cdot)$ and flow variable $u(\cdot)$, instead of consumptions. The objective becomes

$$
C(\sigma)=\int_{0}^{\infty} e^{-(r+\pi) t}(\pi c(E(t))+r c(u(t))) d t+\sum_{i} e^{-(r+\pi) m_{i}} \gamma
$$

where $c:(-\infty, 0) \rightarrow \mathbb{R}$ denotes the inverse of the utility function:

$$
c(v)=-\log (-v) / \rho
$$

The incentive constraint (2) becomes

$$
E(t) \geq \int_{t}^{s} e^{-r(x-t)} e^{-\rho w} r u(x) d x+e^{-r(s-t)} E(s), \forall s \in\left(t, m_{i+1}\right),
$$

since CARA utility implies that $v\left(c^{U}(x)+w\right)=e^{-\rho w} v\left(c^{U}(x)\right)=e^{-\rho w} u(x)$.

\subsection{Scaling}

Our mechanism exhibits a scaling property: if the initial promise $U(0)$ is scaled by $\alpha>0$, then the optimal contract is also scaled by $\alpha$. More formally,

LEMma 1 If $\{(U(t), E(t), u(t)) ; t \geq 0\}$ are optimal utilities for initial promise $U(0)$, then the optimal utilities for initial promise $\alpha U(0)$ are

$$
\{(\alpha U(t), \alpha E(t), \alpha u(t)) ; t \geq 0\}
$$

Alternatively, Lemma 1 states that the consumption of the worker with initial promise $\alpha U(0)$ differs from that of the worker with promise $U(0)$ by a constant, $-\log (\alpha) / \rho$, at all dates and states.

The scaling property in Lemma 1 is related to the fact that CARA utility has no wealth effect. Although a worker with high promised utility consumes (permanently) more than a worker with low promised utility, the level of promised utility does not have an effect on the worker's incentives to conceal earnings. In other words, the incentive constraint (6) holds when all of the utilities are scaled by the same factor. 
Since the incentives to conceal earnings are the same for workers with different promised utilities, the optimal sequence of monitoring dates, $\left\{m_{i} ; i \geq 1\right\}$, is independent of the initial promised utility. Again, no wealth effect implies that the level of promised utility does not change how the worker is monitored, even if it does change the worker's consumption.

\subsection{Periodicity}

At time 0, the principal knows the true employment status of the agent. After the verification at $m_{1}$, the principal again knows the true employment status. Hence, the continuation problem at $m_{1}$ is the same as the problem at time 0 , except for the "initial" promised utility. The scaling property implies that, if $U\left(m_{1}\right)=\alpha U(0)$, then the optimal utilities from $m_{1}$ forward are scaled by $\alpha$. Thus, starting with a promise $U(0)$, if the principal finds it optimal to monitor the unemployed agent at $m_{1}$, then it must be the case that starting with the promise $\alpha U(0)$ the principal would again find it optimal to monitor at $m_{1}$. Put differently, having monitored the agent at $m_{1}$, the next optimal monitoring period is $2 m_{1}$. We immediately conclude that

Proposition 1 The optimal monitoring is periodic, i.e., $m_{i}=i m_{1}$ for all $i \geq 1$.

To understand the intuition for the periodic monitoring, consider policies where the interval between verifications is either increasing or decreasing over time. First, it is suboptimal for the planner to verify more frequently at the beginning. Since the worker starts out unemployed, he stays unemployed for some duration initially. Frequent verifications early on merely incur unnecessary verification cost. Second, one might think that it is optimal to verify more frequently later since the probability of a long duration of unemployment is small. However, this policy is also suboptimal. The worker's conditional probability of transitioning to employment is independent of how long he has been unemployed. Moreover, because the principal knows the true employment status after each verification, the scaling property implies that from the principal's perspective the worker who was just verified to be unemployed is no different from the worker at time zero. Thus the interval between consecutive monitoring periods is a constant. 
While we have established that the optimal monitoring is periodic, finding the optimal periodicity is difficult. To determine the optimal $m_{1}$ we must first determine the optimal utilities in the intervals $\left[0, m_{1}\right],\left[m_{1}, 2 m_{1}\right]$, etc. Toward this end, we break the principal's problem into two steps. First, assume that $m_{1}$ is exogenous and the principal learns the agent's employment status at dates $m_{1}, 2 m_{1}$, etc. Given $m_{1}$, the principal solves for the endogenous utility paths in $\left[0, m_{1}\right],\left[m_{1}, 2 m_{1}\right]$, etc. Second, the principal chooses $m_{1}$ optimally. We analyze the first step in the next section and the second step in section 5 .

\section{Optimal Unemployment Insurance with Exogenous Monitoring}

Given the simplification in Section 3, we now present the features of the optimal unemployment insurance scheme. For a given $m_{1}$, we first formulate the optimal control problem in Section 4.1. This allows us to analyze the time paths of the variables of interest. We then describe some features of the continuation utilities $E(\cdot)$ and $U(\cdot)$ in Section 4.2 and use these features to illustrate the employment tax in Section 4.3 and unemployment benefits in Section 4.4. Finally, in Section 4.5 we use the Pontryagin Minimum Principle to explicitly characterize $E(\cdot)$ and $U(\cdot)$.

\subsection{Optimal Control}

We formulate the principal's problem for interval $\left[0, m_{1}\right]$ as one of optimal control. Our analysis for $\left[0, m_{1}\right]$ applies to other intervals as well.

First, we rewrite the constraints recursively. The promise-keeping constraint (1) is equivalent to the differential equation:

$$
U^{\prime}(t)=r(U(t)-u(t))+\pi(U(t)-E(t)) .
$$

On the right side of the differential equation, the first term is the rate of change of $U$ when there is no uncertainty (i.e., when there is no transition to employment), and the second term captures the additional rate of change due to uncertainty. 
The incentive constraint (6) is equivalent to the following differential inequality:

$$
r\left(v\left(c^{U}(t)+w\right)-v\left(c^{E}(t)\right)\right)+E^{\prime}(t) \leq 0 .
$$

That is, the short term benefit that the agent gets from fraud, $r\left(v\left(c^{U}(t)+w\right)-v\left(c^{E}(t)\right)\right)$, is offset by lower continuation utility he receives after he delays the employment report. Note that $E(\cdot)$ could have downward jumps: when $E(t)>\lim _{s \downarrow t} E(s)$, we interpret the discontinuity as $E^{\prime}(t)=-\infty$, and the differential inequality (7) still holds under this interpretation. Introducing a slack variable $\mu(t) \geq 0$, we may rewrite (7) as

$$
E^{\prime}(t)=r E(t)-e^{-\rho w} r u(t)-\mu(t)
$$

In Lemma B.1 in Appendix C, we show that the above differential equation and inequality are equivalent to (1) and (6).

Second, the scaling property implies that the cost function $C(\cdot)$ satisfies

$$
C(\alpha U)=C(U)-\log (\alpha) / \rho .
$$

Recalling the definition of $c(\cdot)$ in $(5)$, we rewrite $C(U)$ as

$$
C(U)=C(|U|(-1))=C(-1)-\log (-U) / \rho \equiv \psi+c(U),
$$

where $\psi \equiv C(-1)$ is the cost of private information: it is the one-time cost that the principal is willing to pay to permanently remove private information from the model.

With $\psi+c\left(U\left(m_{1}\right)\right)$ as the continuation cost at $m_{1}$, we rewrite the principal's problem as one of optimal control with a convex objective and linear constraints.

$$
\begin{array}{ll}
\min _{\substack{u(t), U(t), E(t), 0 \leq t \leq m_{1}}} & \int_{0}^{m_{1}} e^{-(r+\pi) t}(\pi c(E(t))+r c(u(t))) d t+e^{-(r+\pi) m_{1}}\left(\gamma+\psi+c\left(U\left(m_{1}\right)\right)\right) \\
\text { subject to } & U^{\prime}(t)=(r+\pi) U(t)-\pi E(t)-r u(t), \\
E^{\prime}(t) & =r E(t)-e^{-\rho w} r u(t)-\mu(t), \\
E(t) & \geq U(t), \\
U(0) & \text { is given. }
\end{array}
$$




\subsection{Continuation Utilities}

The continuation utilities $E(\cdot)$ and $U(\cdot)$ help us uncover the consumption paths for the employed and the unemployed. We focus on the properties of $E(\cdot)$ and $U(\cdot)$ in $\left[0, m_{1}\right]$; those in other monitoring cycles can be obtained by scaling (see Lemma 1).

We demonstrate five properties:

(i) $E(t)>E(s)$ for $t<s \leq m_{1}$.

(ii) $E(t)>U(t)$ for all $t<m_{1}$.

(iii) $E\left(m_{1}\right)=U\left(m_{1}\right)$.

(iv) $E(\cdot)$ jumps up immediately after $m_{1}$.

(v) $U(\cdot)$ declines over time.

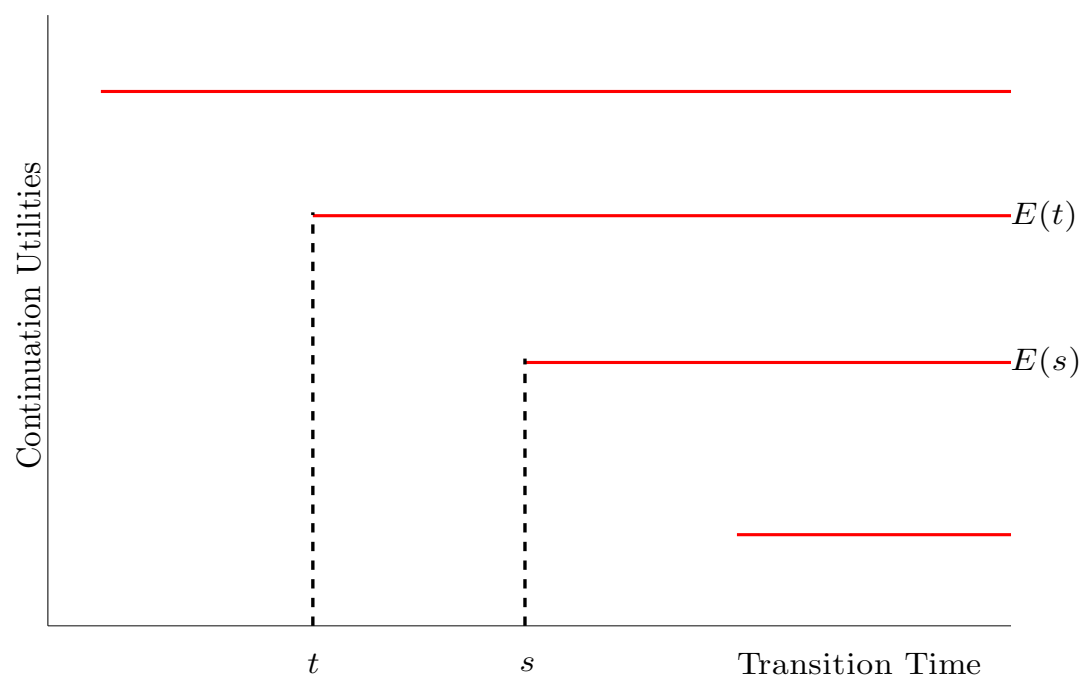

Figure 1: Lower payoff for late reporters $(E(t)>E(s)$ for $t<s)$

Property (i) states that the payoff to a worker who reports the transition to employment earlier is higher than the payoff to one who reports the transition later. The worker who transitions to employment at $t$ but commits fraud consumes $c^{U}(t)+w$ at $t$, whereas the worker who tells the truth consumes $c^{E}(t)$. It is intuitive that $c^{E}(t)<c^{U}(t)+w$; otherwise 
deterring fraud would not be an issue. In terms of utilities, $E(t)<e^{-\rho w} u(t)$. Incentive compatibility (11) requires that delaying the report yields a lower payoff (see Figure 1). Thus, $E(t)>E(s)$ within a monitoring cycle.

For property (ii), recall that restriction (12) imposes $E(t)$ must be greater than or equal to $U(t)$. If the agent who transitions to employment before $m_{1}$ is offered the same payoff as the agent who remains unemployed, then the employed agent will claim to be unemployed and consume more than the unemployed agent. He can continue cheating until the verification period $m_{1}$ (see Figure 2). Thus, within a monitoring cycle, $E(t)$ must be greater than $U(t)$.

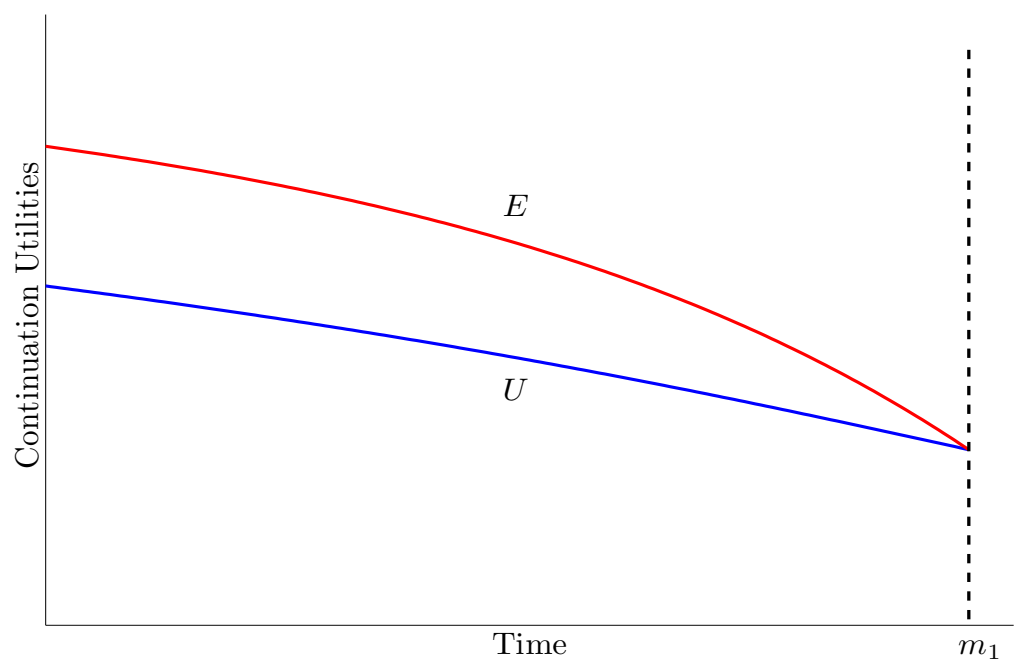

Figure 2: Continuation utilities $E(\cdot)$ and $U(\cdot)$ in $\left[0, m_{1}\right]$.

To understand (iii), note that the true employment status is revealed at $m_{1}$, so the principal does not face an incentive problem at that instant. Hence, there is no reason to reward the (lucky) agent who transitioned to employment at $m_{1}$ relative to the (unlucky) agent who remains unemployed i.e., no reason to set $E\left(m_{1}\right)>U\left(m_{1}\right)$. Thus, $E\left(m_{1}\right)=$ $U\left(m_{1}\right)$. (Again, recall restriction (12): $E(t) \geq U(t)$ for all $t$.)

Property (iv) states that $U\left(m_{1}\right)=E\left(m_{1}\right)<E\left(m_{1}+\right)$, where $E\left(m_{1}+\right)$ is the utility for a worker who is unemployed at $m_{1}$ but transitions to employment immediately after $m_{1}$, i.e., $E\left(m_{1}+\right)=\lim _{t \downarrow m_{1}} E(t)$ (see Figure 3). Suppose, to the contrary, that $U\left(m_{1}\right)=E\left(m_{1}+\right)$. 
Then incentive compatibility in $\left[m_{1}, 2 m_{1}\right]$ would be violated because the worker employed immediately after $m_{1}$ can claim to be unemployed and consume more than the employed until the next verification period, $2 m_{1}$. Note that if there is no verification at date $t$, then an upward jump in $E(\cdot)$ violates the incentive constraint: a worker who transitions to employment prior to $t$ would benefit from delaying the employment report. At the moment of verification, however, the worker cannot delay the employment report since the true employment status is revealed.

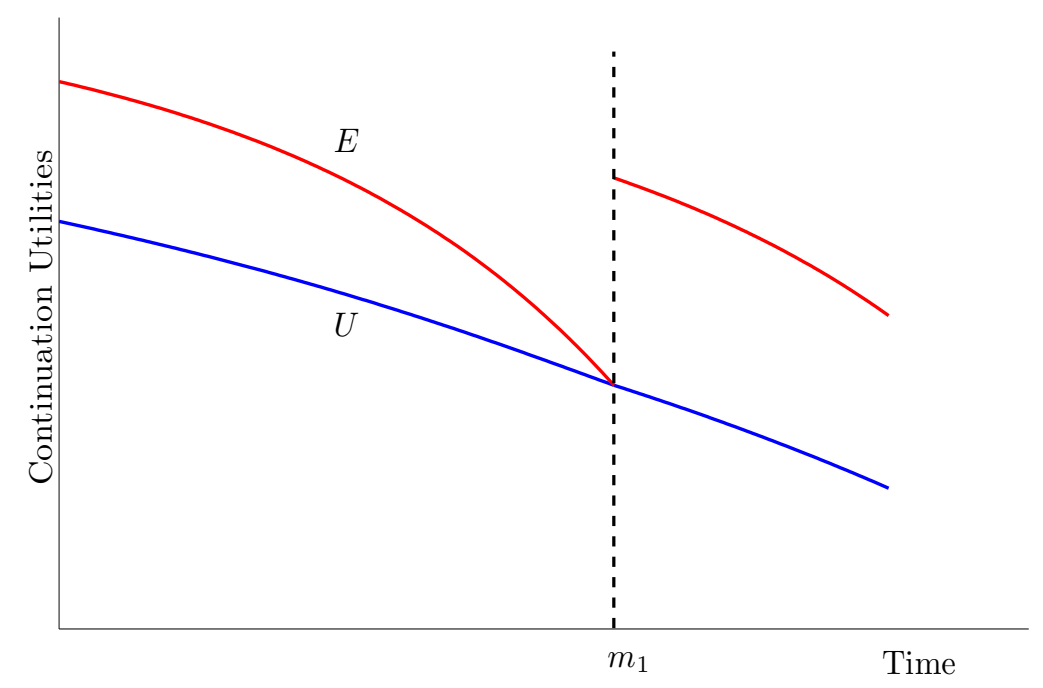

Figure 3: Continuation utility $E(\cdot)$ is nonmonotonic.

To understand why $U(\cdot)$ declines, suppose $U\left(m_{1}\right)>U(0)$. Then lowering $U\left(m_{1}\right)$ has two benefits. First, the unemployed agent's continuation utility path is flatter, which implies better insurance for the unemployed. Second, lower $U\left(m_{1}\right)$ (and $\left.E\left(m_{1}\right)\right)$ reduces $E^{\prime}(\cdot)$, generating stronger incentives to deter fraud. In addition, $U(\cdot)$ can never jump. Because $U(\cdot)$ is the promised utility to the unemployed agent, any jump in $U(\cdot)$ would violate the promise-keeping constraint. 


\subsection{Employment Tax}

Here we examine the consumption allocated to the agent who reports employment earlier relative to the consumption for the agent who reports it later. Recall that $E(t)>E(s)$ within a monitoring cycle and the continuation utility $E(\cdot)$ jumps up after verification. Since employment is an absorbing state, any agent who reports a transition to employment at $t$ is allocated constant consumption $c^{E}(t)$ forever and is not monitored. Thus, $E(t)$ maps into $c^{E}(t)$ instant by instant and, hence, $c^{E}(t)>c^{E}(s)$ within a monitoring cycle. Furthermore, the consumption for the agent who reports the transition to employment immediately after $m_{1}$ is higher than that for the employed agent at $m_{1}$ (see Figure 4 ).

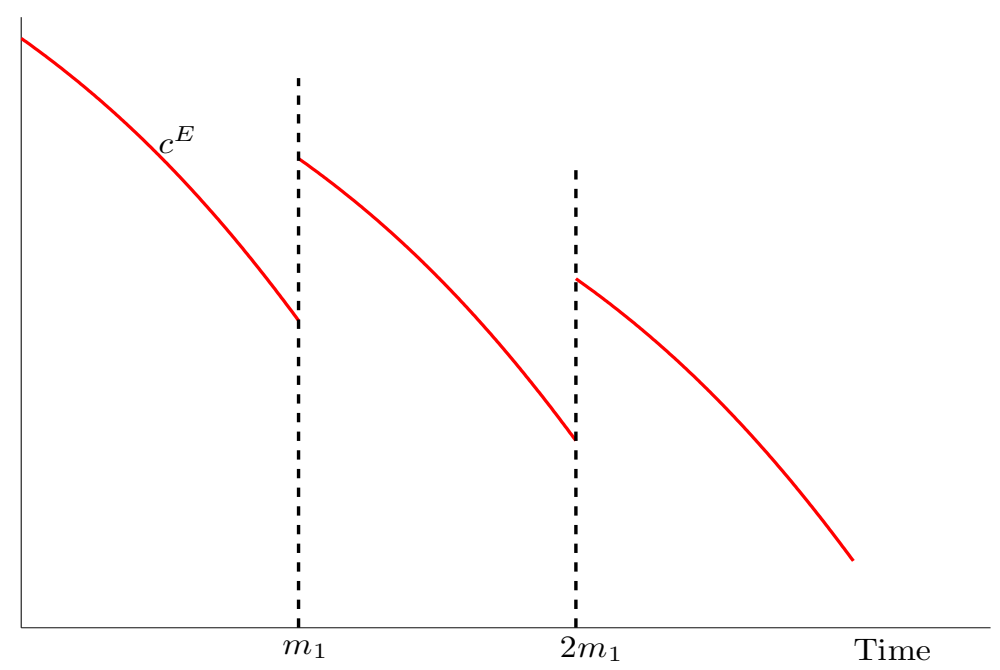

Figure 4: Permanent consumption for workers who transition to employment in different periods

The nonmonotonicity is closely related to the way incentives are provided in our model. Within a cycle, the principal does not monitor, and relies exclusively on consumption distortions to induce truth-telling: $c^{E}$ must fall sufficiently fast for the worker not to postpone his report of employment. At $m_{1}, c^{E}$ falls to a level such that the agent is indifferent between transitioning to employment and remaining unemployed. The principal can perfectly insure the agent against the unemployment shock at $m_{1}$ because the true 
employment status is revealed. Immediately after $m_{1}$, the principal treats the worker employed right after $m_{1}$ better than the worker employed at $m_{1}$. This is because the worker who transitions to employment after $m_{1}$ can commit fraud until the next monitoring period, while the worker who transitions to employment at $m_{1}$ cannot commit fraud. Hence, the principal must offer the former a higher permanent consumption to induce truth-telling.

The difference between wage $w$ and consumption $c^{E}$ can be interpreted as an employment tax. Our contract implies that within a verification cycle, the employment tax for late reporters is higher than that for the early reporters. However, unlike the existing unemployment insurance literature, the employment tax is nonmonotonic: it decreases immediately following verification.

\subsection{Unemployment Benefits}

Unlike the case where $c^{E}(t)$ maps into $E(t)$ at every instant, $c^{U}(t)$ is not pinned down at every instant by $U(t)$, since the unemployed agent is not fully insured. Instead, the path of $c^{U}(\cdot)$ in $\left[0, m_{1}\right]$ requires knowledge of the entire path of $U(\cdot)$ in the interval. We obtain the entire trajectories of $c^{U}(\cdot)$ and $U(\cdot)$ after solving (9) in Section 4.5. However, monotonicity of $U(\cdot)$ in Section 4.2 suggests that $c^{U}(\cdot)$ declines with unemployment duration. As in Hopenhayn and Nicolini (1997), our contract implies that the unemployment benefit $c^{U}$ eventually reaches an arbitrarily low level with positive probability. ${ }^{6}$

Figure 5 shows that the unemployment benefits jump down at the verification period. To understand the jump, we argue that it is optimal for the principal to set $u(t)$ above $u\left(m_{1}\right)$ when $m_{1}-t>0$ is small. Doing this relaxes the incentive constraint at time $t$, as the following variational argument shows. The promise-keeping constraint at $m_{1}-\delta$, for a small positive $\delta$, is

$$
\begin{aligned}
U\left(m_{1}-\delta\right) & =r \delta u\left(m_{1}-\delta\right)+e^{-r \delta}\left[(\pi \delta) E\left(m_{1}\right)+(1-\pi \delta) U\left(m_{1}\right)\right] \\
& =r \delta u\left(m_{1}-\delta\right)+e^{-r \delta} U\left(m_{1}\right)
\end{aligned}
$$

\footnotetext{
${ }^{6}$ In contrast to Hopenhayn and Nicolini (1997) and our paper, Pavoni (2007) imposes an exogenous lower bound on promised utility and shows that the optimal benefits decrease with the duration of unemployment, but remain constant after the promised utility reaches the lower bound. Alvarez-Parra and Sanchez (2009) show a similar result in a model with an endogenous lower bound on promised utility.
} 


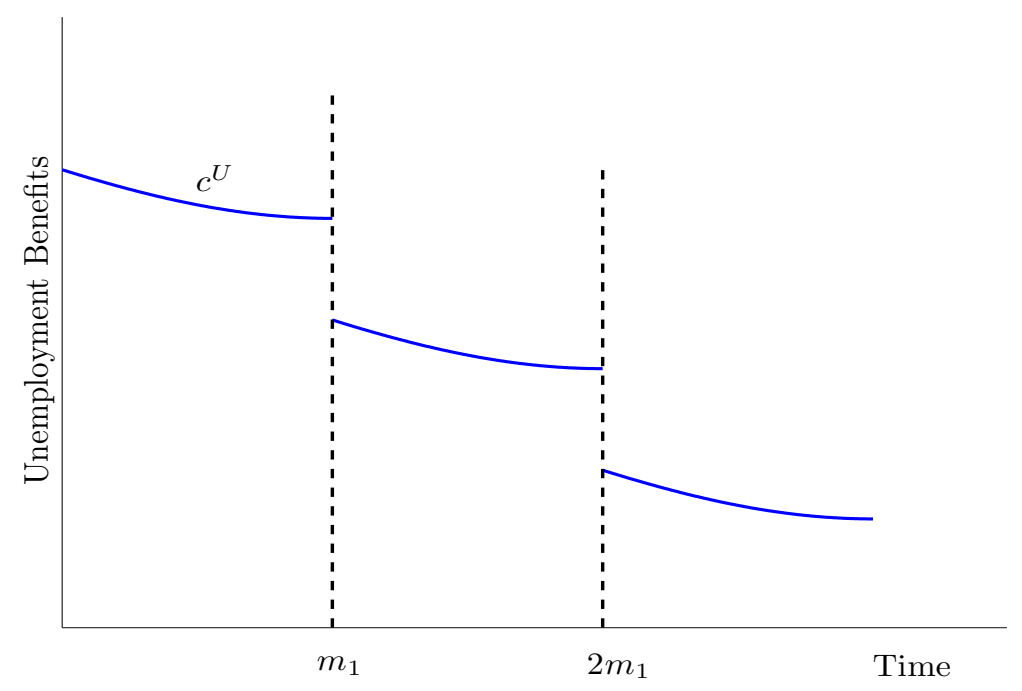

Figure 5: Consumption for the Unemployed

where the second equality uses the aforementioned property $E\left(m_{1}\right)=U\left(m_{1}\right)$. The incentive constraint at $m_{1}-\delta$ is

$$
E\left(m_{1}-\delta\right) \geq r \delta e^{-\rho w} u\left(m_{1}-\delta\right)+e^{-r \delta} E\left(m_{1}\right)
$$

Suppose $u\left(m_{1}-\delta\right)=u\left(m_{1}\right)$. Then the principal can maintain the promise-keeping constraint but relax the incentive constraint by increasing $u\left(m_{1}-\delta\right)$ and decreasing $u\left(m_{1}\right)$. Specifically, consider the variation

$$
\tilde{u}\left(m_{1}-\delta\right)=u\left(m_{1}-\delta\right)+e^{-r \delta} \epsilon, \quad \tilde{u}\left(m_{1}\right)=u\left(m_{1}\right)-\epsilon, \quad \tilde{E}\left(m_{1}\right)=E\left(m_{1}\right)-r \delta \epsilon .
$$

Because the unemployed worker's consumption after $m_{1}$ remains unchanged, his continuation utility at $m_{1}$ is $\tilde{U}\left(m_{1}\right)=U\left(m_{1}\right)-r \delta \epsilon$, which is equal to $\tilde{E}\left(m_{1}\right)$. Therefore, the promise-keeping constraint $U\left(m_{1}-\delta\right)=r \delta \tilde{u}\left(m_{1}-\delta\right)+e^{-r \delta} \tilde{U}\left(m_{1}\right)$ still holds, and the incentive constraint is relaxed:

$$
\begin{aligned}
r \delta e^{-\rho w} \tilde{u}\left(m_{1}-\delta\right)+e^{-r \delta} \tilde{E}\left(m_{1}\right) & =r \delta e^{-\rho w} u\left(m_{1}-\delta\right)+e^{-r \delta} E\left(m_{1}\right)-\left(1-e^{-\rho w}\right) r \delta \epsilon \\
& <r \delta e^{-\rho w} u\left(m_{1}-\delta\right)+e^{-r \delta} E\left(m_{1}\right) .
\end{aligned}
$$

Starting from $u\left(m_{1}-\delta\right)=u\left(m_{1}\right)$, the additional cost of consumption incurred by this 
variation is second order, but the effect on incentive constraint is first order. Hence the principal always chooses $u(t)$ above $u\left(m_{1}\right)$ when $t$ is close to (but below) $m_{1}$.

We summarize these findings in the following proposition. The proof is in Appendix C.

Proposition 2 The unemployment benefit, $c^{U}(\cdot)$ is monotonically decreasing with unemployment duration, with downward jumps at verification, while $c^{E}(\cdot)$ is nonmonotonic: it decreases between verifications with upward jumps immediately after verification.

Unemployment insurance systems in many countries feature benefits schemes similar to the one in Proposition 2. For example in Spain, workers receive a replacement rate of 70 percent for the first 6 months of unemployment, 60 percent for the next 18 months, and a minimum payment thereafter.

\subsection{Pontryagin Minimum Principle}

We construct a solution to the optimal control problem (9) in which the incentive constraint (11) binds (i.e., $\mu(t)=0$ ) for all $t<m_{1}$. The problem faced by the principal is to choose an initial state $E(0)$ and a time path $u(\cdot)$ to minimize the cost in (9), given $U(0)$. The promise-keeping and incentive constraints (10) and (11) then imply a time path $(U(\cdot), E(\cdot))$ for continuation utilities. One way to think about this problem is to think of choosing $u(t)$ at each date, given the values of $U(t)$ and $E(t)$ that have been attained by that date. The principal faces a tradeoff between the current-period cost and the cost of delivering continuation utilities. Hence, she needs to set "prices", $\Phi$ and $\lambda$, on increments to the continuation utilities $U$ and $E$. Because it is costly for the principal to maintain a low $E$ as a threat, it must be the case that $\lambda \leq 0$. Moreover, we have argued in Section 4.2 that $E(t) \geq U(t)$ is slack except at $m_{1}$, so we impose only the constraint $E\left(m_{1}\right)=U\left(m_{1}\right)$.

A central construct in the optimal control problem is the current value Hamiltonian $\mathscr{H}$ defined by

$$
\mathscr{H}=\pi c(E(t))+r c(u(t))+\Phi(t)((r+\pi) U(t)-\pi E(t)-r u(t))+\lambda(t)\left(r E(t)-e^{-\rho w} r u(t)\right),
$$

which is just the sum of current-period cost and the rate of increase in continuation utilities valued at $\Phi(t)$ and $\lambda(t)$. An optimal allocation must minimize $\mathscr{H}$ at each date $t$. 
The first-order condition for minimizing $\mathscr{H}$ with respect to $u$ is

$$
c^{\prime}(u)=\Phi+e^{-\rho w} \lambda
$$

The left-hand side is the marginal cost of today's utility, while the right-hand side is the marginal cost of starting with higher continuation utility $U$ tomorrow, offset by the benefit of a slacker incentive constraint (it is a benefit because $\lambda \leq 0$ ). The utility $u$ must be chosen to equalize the costs at each date.

The prices $\Phi$ and $\lambda$ must satisfy

$$
\begin{aligned}
\Phi^{\prime}(t) & =(r+\pi) \Phi-\frac{\partial \mathscr{H}}{\partial U}=0, \\
\lambda^{\prime}(t) & =(r+\pi) \lambda-\frac{\partial \mathscr{H}}{\partial E}=\pi\left(\Phi-c^{\prime}(E)+\lambda\right),
\end{aligned}
$$

at each date $t$ if $(u(\cdot), U(\cdot), E(\cdot))$ is an optimal path. Equation (14) implies that $\Phi(t)$ is a constant. Moreover, since multiplier $\Phi(0)$ is the marginal cost of $U(0)$, we have

$$
\Phi=C^{\prime}(U(0))=-(\rho U(0))^{-1}>0 .
$$

Since the planner can choose $E(0)$ freely,

$$
\lambda(0)=0
$$

At $m_{1}$, the shadow prices $\Phi$ and $\lambda\left(m_{1}\right)$ must satisfy

$$
\begin{aligned}
\Phi & =-\kappa+c^{\prime}\left(U\left(m_{1}\right)\right), \\
\lambda\left(m_{1}\right) & =\kappa,
\end{aligned}
$$

where $e^{-(r+\pi) m_{1}} \kappa$ is the multiplier on the constraint $E\left(m_{1}\right)=U\left(m_{1}\right)$. Since the principal's problem is convex, these conditions (13-18) are both necessary and sufficient for a minimum.

When (11) holds as equality, the states $(U, E)$ and the costate $\lambda$ satisfy differential equations:

$$
\begin{aligned}
U^{\prime}(t) & =(r+\pi) U-\pi E-r u, \\
E^{\prime}(t) & =r E-r e^{-\rho w} u, \\
\lambda^{\prime}(t) & =\pi\left(\Phi-c^{\prime}(E)+\lambda\right) .
\end{aligned}
$$


The ODE system contains three variables and would be difficult to analyze in a general context. However, we can solve (20) and (21) regardless of (19), because neither (20) nor (21) relies on $U$. Once (20) and (21) are solved, it is easy to solve (19). Formally,

LEMMA 2 If (20) and (21) hold, then (19) holds if and only if

$$
\Phi U(t)+\lambda(t) E(t)+\rho^{-1}=0, \quad \forall t \in\left[0, m_{1}\right] .
$$

To solve the reduced ODE system, (20) and (21), we need two boundary conditions. The first is $(16), \lambda(0)=0$. The second cannot be a value for $E(0)$, as $E(0)$ is endogenous and unknown a priori. We obtain the second boundary condition, $E\left(m_{1}\right)=-\rho^{-1}\left(\Phi+\lambda\left(m_{1}\right)\right)^{-1}$, from $E\left(m_{1}\right)=U\left(m_{1}\right)$ and equation $(22)$.

The following lemma shows that these two boundary conditions pin down a unique solution curve for the system (20) and (21). Figure 6 shows the phase diagram. That $\lambda<0$ implies that the incentive constraint binds for all $t<m_{1}$.

LEMma 3 For any $m_{1}>0$, there is a unique initial condition $E(0)$ such that the solution starting at $(\lambda(0)=0, E(0))$ satisfies $E\left(m_{1}\right)=-\rho^{-1}\left(\Phi+\lambda\left(m_{1}\right)\right)^{-1}$.

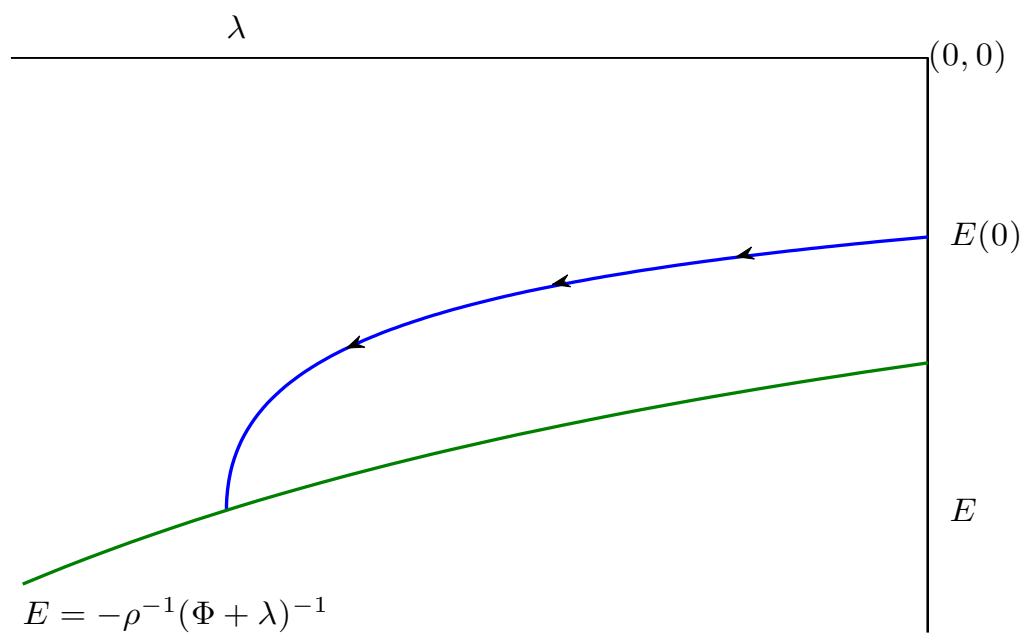

Figure 6: Phase Diagram for $(\lambda, E)$. 


\section{Optimal Monitoring}

Until this point, we have taken $m_{1}$ as exogenous. In this section, we characterize the optimal choice of $m_{1}$. The tradeoff in choosing $m_{1}$ is as follows. Monitoring more frequently implies higher verification cost, but the principal can provide better insurance: the consumption path for the unemployed is similar to that for the employed. Monitoring less frequently implies lower verification cost but worse insurance.

For any $m_{1}>0$, denote the minimized cost in $(9)$ as $\mathscr{C}\left(m_{1}\right)$; that is,

$$
\mathscr{C}\left(m_{1}\right)=\int_{0}^{m_{1}} e^{-(r+\pi) t}(\pi c(E(t))+r c(u(t))) d t+e^{-(r+\pi) m_{1}}\left(\gamma+\psi+c\left(U\left(m_{1}\right)\right)\right) .
$$

Intuitively, delaying monitoring (i.e., a small increase in $m_{1}$ ) saves the principal both the cost of monitoring and the cost of (after-monitoring) consumptions, because the payment of $\gamma+\psi+c\left(U\left(m_{1}\right)\right)$ is postponed. By doing so, however, the principal must maintain the consumptions $c(E(\cdot))$ and $c(u(\cdot))$ for a longer duration. Subtracting the benefit from the cost (algebraic details in Appendix C) yields

$$
\mathscr{C}^{\prime}\left(m_{1}\right)=e^{-(r+\pi) m_{1}}\left(r \rho^{-1} \log \left(\frac{\Phi+e^{-\rho w} \lambda\left(m_{1}\right)}{\Phi+\lambda\left(m_{1}\right)}\right)-(r+\pi)(\gamma+\psi)\right) .
$$

Thus, the first-order condition for $m_{1}$ is

$$
r \rho^{-1} \log \left(\frac{\Phi+e^{-\rho w} \lambda\left(m_{1}\right)}{\Phi+\lambda\left(m_{1}\right)}\right)=(r+\pi)(\gamma+\psi) .
$$

Proposition 3 The optimal $m_{1}$ is the unique solution to (23). That is, (23) is both necessary and sufficient for the minimum of $\mathscr{C}\left(m_{1}\right)$.

REMARK 1 Although our analysis relies on an undetermined parameter $\psi$, the parameter can be uniquely pinned down by a fixed-point condition that the actual cost function at time zero must equal the conjectured function $\psi+c(U(0))$. Further details are in Appendix $C$.

REMARK 2 Our analytical results rely on the assumption of CARA preferences. Unlike the CARA case where the length of the monitoring cycle is independent of history, the cycle length in the CRRA case depends on the worker's continuation utility. However, most of the main features of the optimal contract remain valid even if the worker has CRRA preferences. We demonstrate this through a numerical example in Fuller, Ravikumar, and Zhang (2013). 


\section{Quits}

Another type of fraud that could arise in our model is quits. An agent in our model could transition to employment in period $t$, claim to be unemployed until almost $m_{1}$, and then quit to become unemployed at $m_{1}$. The verification at $m_{1}$ would not reveal him to be a cheater. Thus, quitting is possible in our model.

Our mechanism guarantees that the agent does not commit such a fraud. The continuation utilities $E(\cdot)$ and $U(\cdot)$ are such that the agent is indifferent between reporting the transition immediately and delaying it to the next period. By following the path above and quitting at $m_{1}$, he becomes truly unemployed, is subject to the stochastic arrival rate of employment opportunity, and is worse off.

Hopenhayn and Nicolini (2009) examine a model where quits cannot be distinguished from layoffs and the only fraudulent behavior is quits. In their model, the employment status is observable and non-absorbing, and disutility from working is greater than that from searching for employment. Employed agents might want to opportunistically quit their job, enjoy more leisure, and collect unemployment benefits. To discourage quits, the principal offers (i) higher consumption to the employed workers who stay on the job longer and (ii) more generous benefits to unemployed workers with longer employment spells, as quitters have shorter employment spells on average. In our model, the utility functions for the unemployed worker and the employed worker are the same, and employment status is private information. Since employment is an absorbing state, quitting as considered in Hopenhayn and Nicolini (2009) cannot arise in our model. The potential reason for quitting in our model is to cover up the fraudulent collection of unemployment benefits before the verification period. Our optimal mechanism provides incentives for the agent not to delay reporting his transition to employment and not to conceal his earnings.

While overpayment due to quits is more than the overpayment due to insufficient search and job refusals, it is small relative to the overpayment due to concealed earnings (see Table 1). Our mechanism deters fraud due to both concealed earnings and quits. 


\section{Stochastic Verification}

Our monitoring mechanism in the previous sections was restricted to deterministic verification. Here we consider a more general mechanism where the principal verifies randomly after receiving the unemployment report. Conditional on the unemployment report at $t$, the principal chooses the monitoring Poisson rate $p(t) \geq 0$. That is, over a period of length $d t$, the principal monitors with probability $p(t) d t$ and she does not monitor with probability $1-p(t) d t$. (Since our model is in continuous time, $p(t)$ is not the monitoring probability.)

We assume that if a worker is monitored and caught cheating, he has to pay a finite penalty forever. With infinite penalty, an arbitrarily small monitoring probability would deliver the full-information constant consumption. In our model, if the principal can choose any finite penalty between 0 and $\phi>0$, he would always choose $\phi$. Henceforth, we assume that the finite penalty is $\phi$ units of the consumption good, forever.

Similar to (10) and (11), the promise-keeping constraint and incentive constraint are

$$
\begin{aligned}
& U^{\prime}=r(U-u)-\pi(E-U)-p(\tilde{U}-U), \\
& E^{\prime} \leq r E-r e^{-\rho w} u-p\left(e^{\rho \phi}-1\right) E
\end{aligned}
$$

where $\tilde{U}$ is the unemployed agent's continuation utility after monitoring. Because the probability that monitoring does not occur in $[0, t)$ is $e^{-\int_{0}^{t} p(s) d s}$, the principal's objective is

$$
\int_{0}^{\infty} e^{-(r+\pi) t-\int_{0}^{t} p(s) d s}(\pi c(E(t))+r c(u(t))+p(t)(\gamma+C(\tilde{U}(t)))) d t .
$$

The principal chooses the utilities $\{U(t), E(t), u(t), \tilde{U}(t) ; t \geq 0\}$ and the arrival rates of monitoring $\{p(t) ; t \geq 0\}$ to minimize (26) subject to $(24),(25)$, and the constraint $E(t) \geq$ $U(t), \forall t \geq 0$.

Since the penalty for a worker with high promised utility is the same as that for a worker with low promised utility, we obtain a scaling property similar to the one in Section 3.1. Thus, the incentives to conceal earnings are the same for workers with different promised utilities. Similar to our model with deterministic verification, we show in Proposition 4 that the optimal stochastic verification mechanism consists of cycles. See Appendix D for the proof. 
Proposition 4 There exists an $N>0$ such that the principal monitors the unemployed with a constant arrival rate $p>0$ if and only if $t \geq N$. Before $N$, the time path $(U(\cdot), E(\cdot))$ converges to the 45-degree line; after $N$, it moves along the 45-degree line toward $(-\infty,-\infty)$ until the agent is randomly drawn to be verified. After the verification, $(U, E)$ jumps to a new state $(\tilde{U}, \tilde{E})$ and a new cycle starts.

The unemployed worker is in one of two states: (i) not monitored (i.e., $p(t)=0$ ) or (ii) randomly drawn to be monitored (i.e., $p(t) \equiv p>0$ ). Within each cycle, an unemployed worker is initially in the not-monitored state. He is moved to the random monitoring state if the duration of his unemployment report exceeds the threshold $N$. If he is randomly drawn to be monitored, then he is moved to the not-monitored state after being monitored, and a new cycle begins. While the date of monitoring is stochastic, the threshold duration is not. That is, within each cycle, the principal guarantees that the worker will not be monitored until the threshold duration is reached, similar to the deterministic verification case.

The intuition for why the worker is not monitored before the threshold duration is as follows. The Unemployment Insurance agency has access to two instruments: tax/subsidy and monitoring. Recall that at verification the true employment status is revealed, and $E$ is reset to a level such that its shadow price is zero, which means that, immediately after monitoring, the employment tax can be varied at no cost. The cost of the tax/subsidy instrument is lower than the cost of monitoring, $\gamma>0$, immediately after monitoring, and remains so until some threshold unemployment duration is reached. Hence, it is optimal to use only the tax/subsidy instrument for the provision of incentives before the threshold.

REMARK 3 The absence of verification until a threshold duration is unlikely to be robust to other types of penalties. For instance, in Popov (2009) there is an exogenous lower bound on the worker's continuation utility and a worker who is caught cheating is pushed to this lower bound. So the penalty for a worker with high continuation utility is larger than that for a worker with low continuation utility. With hidden i.i.d. income, he shows that the verification probability is always positive.

The stochastic monitoring mechanism clearly dominates the deterministic mechanism characterized in Section 5. To see this, consider a stochastic monitoring scheme in which 
the arrival rate of monitoring is higher than $p$ for workers in the random monitoring state. Denote this higher arrival rate as $\tilde{p}$. Proposition 4 implies that $\tilde{p}$ is suboptimal. By continuity, the limiting scheme as $\tilde{p} \rightarrow \infty$ should also be suboptimal. This limiting scheme is exactly the deterministic monitoring mechanism.

We argue below that the key insights on the use of tax/subsidy and monitoring instruments in the suboptimal deterministic mechanism are nearly identical to the insights from the optimal stochastic mechanism. We describe in detail the similarities and differences between the implications of the two mechanisms.

\subsection{Comparison of Monitoring with the Deterministic Case}

First, both the stochastic and deterministic mechanisms have the feature that monitoring does not occur before a threshold unemployment duration; $m_{1}$ in the deterministic case and $N$ in the stochastic case. These thresholds, however, could be different; i.e., in general $m_{1} \neq N$.

Second, both mechanisms feature cycles. In the deterministic case, after $m_{1}$ a new cycle begins, with exactly the same length as the previous cycle. Similarly, in the stochastic case, after monitoring occurs a new cycle begins and verification does not occur again before the threshold $N$ is reached. The exact date when the monitoring occurs in the stochastic case is random. This is because, after $N$ monitoring arrives according to a Poisson process and, hence, the exact length of each cycle depends on when the worker is actually verified. As in the deterministic case, however, the value of $N$ is the same in each cycle.

\subsection{Comparison of Tax/Subsidy with the Deterministic Case}

Consumptions in the stochastic monitoring case are similar to those in the deterministic case. Within each cycle, before the threshold $N$, the patterns of consumption are identical to $\left(c^{E}, c^{U}\right)$ in Figures 4 and 5 . After $N$, if a worker is monitored and verified to be truly unemployed, then the unemployment benefits jump down, as in the deterministic case.

The only difference is that in the deterministic case, continuation utilities and consumptions are reset when the threshold $m_{1}$ is reached. In the stochastic case, after the thresh- 
old $N$ and before the monitoring actually arrives, continuation utilities and consumptions smoothly decline with the duration of unemployment. The decreasing continuation utilities and the monitoring (and finite punishment) jointly provide incentives for truth telling; the worker is indifferent between reporting a job offer and committing fraud.

\subsection{Quantitative Analysis}

In this section, we perform a quantitative exercise similar to Hopenhayn and Nicolini (1997). We let the agents in our model face a stylized version of the U.S. unemployment insurance system. We calibrate the model to match the observed rate of concealed earnings fraud. We then compute the gain to switching to the optimal mechanism in our model.

To perform this exercise, we have to add some heterogeneity to our model; otherwise everyone would cheat or no one would cheat, and we would not be able to match the observed rate of concealed earnings fraud. We assume that the workers are heterogeneous in the wages they earn and, hence, the replacement rate for unemployment benefits. Concretely, we assume that the wage distribution is lognormal with parameters $\mu_{w}$ and $\sigma_{w}^{2}$.

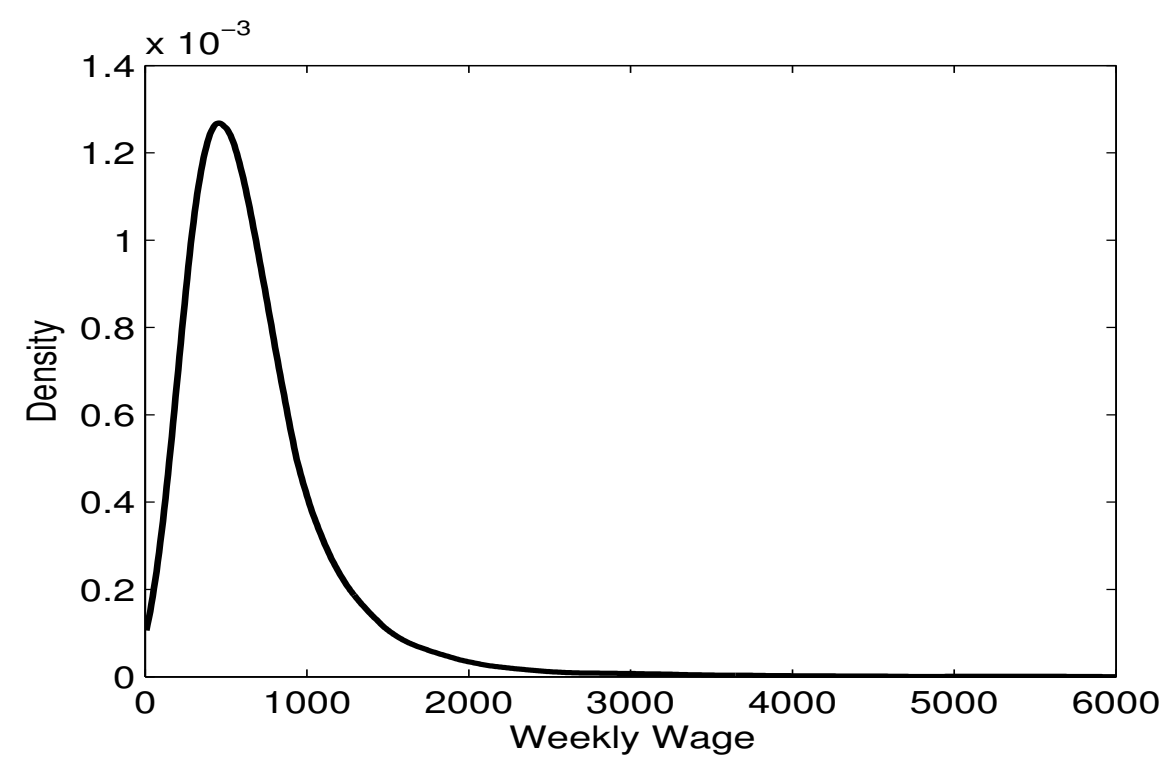

Figure 7: Kernel Density Estimate of Weekly Wages, 2007.

The BAM data provides earnings information for an individual's previous employment 
(the earnings that determine the amount of UI benefits for the individual). In the 2007 sample of BAM data, the mean weekly wage is $\$ 692$ and the coefficient of variation is 0.79 (see Figure 7). Using these data moments, we calibrate $\mu_{w}=6.296$ and $\sigma_{w}^{2}=0.488$. By construction, the earnings in the BAM data are only for those who collect unemployment benefits. Instead of using the BAM data we could use the CPS data on earnings for the entire employed population to calibrate the wage distribution in the model. However, individuals collecting unemployment benefits generally earn less (while employed) than the individuals in the entire employed population. ${ }^{7}$

We calculate the unemployment benefits as a function of wages, again using the BAM 2007 data: $\ln ($ unemployment benefits $)=1.31+0.65 \ln$ (wages).

We assume that the model period is 1 week and that the interest rate $r=0.001$. Since the average duration of unemployment in 2007 is 16.85 weeks, we calibrate the job arrival rate to be $\pi=1 / 16.85$. The monitoring cost $\gamma$ is calibrated as follows. On average, the BAM investigators spend 12.6 hours per case and the average wage of the investigators is $\$ 43$ in 2012 (the only year when such data is available). So, adjusting the average wage to 2007 dollars, we calibrate $\gamma$ to be $\$ 501$. We calibrate the value of absolute risk aversion $\rho$ such that the relative risk aversion for the average wage earner is 2 . Since average wage is $\$ 692$ in our sample, $\rho=2 / 692$.

We then calibrate the probability of monitoring and the penalty in the U.S. system if caught cheating to match two targets: fraction of people committing concealed earnings fraud and fraction of people caught cheating among those committing the fraud.

With CARA preferences, wage heterogeneity is not relevant for matching the two targets, but it is relevant for computing the distribution of initial promised utility in the baseline. In the counterfactual, we take these initial promised utilities as given, calculate the optimal monitoring and benefits, and then compute the cost of delivering the initial promised utilities. The job arrival rate, wage distribution, and penalty are held fixed at the same values as the baseline calibration.

The results imply that, measured in present value, optimal monitoring costs 60 percent

\footnotetext{
${ }^{7}$ The mean weekly wage among employed workers, in the March 2007 CPS, is $\$ 861$ and the coefficient of variation is 1.27 .
} 
less than the current U.S. system. In the optimal contract (averaging across the initial promised utilities), $N=11.64$ weeks. That is, the planner guarantees that monitoring does not occur for roughly the first 12 weeks of the unemployment spell and, thus, reduces the monitoring cost with an efficient use of the monitoring technology.

To determine the magnitude of the gain from switching to the optimal mechanism, suppose that the planner is restricted to use the same amount of resources as the current U.S. system. How much additional utility can the planner deliver to the average worker? The answer is a utility gain equivalent to a $1.55 \%$ more consumption at every date, relative to the U.S. system. This gain arises from two sources: (i) improved consumption smoothing between employed and unemployed states and (ii) reduced monitoring costs or higher consumption on average. The U.S. system spends only 0.24 percent its resources on monitoring the average worker and spends the rest on unemployment benefits (net of wages), but the same resources are allocated differently in the optimal contract: 0.17 percent is spent on monitoring the average worker and the rest is spent on unemployment benefits. Thus, almost all of the gain in our model comes from improved consumption smoothing.

\section{Conclusion}

The most prevalent incentive problem in the U.S. unemployment insurance system is that individuals collect unemployment benefits while being gainfully employed. We examine a model of optimal unemployment insurance where a worker can conceal his employment status and the Unemployment Insurance authority has a technology to verify his employment status. We find that the optimal interval between consecutive monitoring periods is a constant, independent of history. The optimal employment tax is nonmonotonic, increasing between verifications and decreasing immediately after a verification. The optimal unemployment benefits decline with unemployment duration with sharp declines after each verification. Our optimal contract also prevents fraud from quits.

Unemployment insurance in our model is a form of social insurance protecting workers against the risk of job loss. Acemoglu and Shimer (1999, 2000), Shimer and Werning (2008), and Alvarez-Parra and Sanchez (2009) explore another role of unemployment insurance. 
They examine environments with heterogeneous jobs, and unemployment insurance helps the worker wait for the appropriate job. Some jobs have higher productivity than others, but such job opportunities arrive less frequently. Unemployment benefits help workers wait for more productive matches and endure longer unemployment durations. The benefits in these environments affect the aggregate composition of jobs. An interesting direction for future research is to extend our environment to multiple jobs and examine optimal monitoring in the presence of the alternative role of unemployment insurance.

Finally, our model does not include any job retention effort. Incorporating the job retention effort into our model requires employment to be stochastic. If workers can conceal earnings, their hidden income could affect their job retention effort. Analyzing interaction between effort and fraud is another interesting direction for future research. 


\section{References}

Acemoglu, D., And R. Shimer (1999): "Efficient Unemployment Insurance," Journal of Political Economy, 107(5), 893-928.

(2000): "Productivity Gains from Unemployment Insurance," European Economic Review, 44(7), 1195-1224.

Aliprantis, C., And O. Burkinshaw (1990): Principles of Real Analysis, Second Edition. Academic Press, Inc., San Diego, CA, United States.

Alvarez-Parra, F., and J. M. Sanchez (2009): "Unemployment Insurance with a Hidden Labor Market," Journal of Monetary Economics, 56(7), 954-967.

Ashenfelter, O., D. Ashmore, and O. Deschenes (2005): "Do Unemployment Insurance Recipients Actively Seek Work? Evidence from Randomized Trials in Four U.S. States," Journal of Econometrics, 125(1-2), 53-75.

Atkeson, A., and R. E. Lucas (1995): "Efficiency and Equality in a Simple Model of Efficient Unemployment Insurance," Journal of Economic Theory, 66(1), 64-88.

BAILY, M. (1978): "Some Aspects of Optimal Unemployment Insurance," Journal of Public Economics, 10(3), 379-402.

Fuller, D. L., B. Ravikumar, and Y. Zhang (2013): "Unemployment Insurance Fraud and Optimal Monitoring," Working Paper 2012-024C, Federal Reserve Bank of St. Louis.

Gauthier-Loiselle, M. (2011): "Find a Job Now, Start Working Later Does Unemployment Insurance Subsidize Leisure?," Working Paper, Princeton University.

Golosov, M., and A. Tsyvinski (2006): "Designing Optimal Disability Insurance: A Case for Asset Testing," Journal of Political Economy, 114(2), 257-279.

Hansen, G., and A. Imrohoroglu (1992): "The Role of Unemployment Insurance in an Economy with Liquidity Constraints and Moral Hazard," Journal of Political Economy, 100(1), 118-142.

Hopenhayn, H., and J. P. Nicolini (1997): "Optimal Unemployment Insurance," Journal of Political Economy, 105(2), 412-438.

(2009): "Optimal Unemployment Insurance and Employment History," Review of Economic Studies, 76(3), 1049-1070.

Pavoni, N. (2007): "On Optimal Unemployment Compensation," Journal of Monetary Economics, 54(6), 1612-1630.

Popov, L. (2009): "Stochastic Costly State Verification and Dynamic Contracts," Working Paper, University of Virginia. 
Ravikumar, B., And Y. Zhang (2012): "Optimal Auditing and Insurance in a Dynamic Model of Tax Compliance," Theoretical Economics, 7(2), 241-282.

Setty, O. (2011): "Optimal Unemployment Insurance with Monitoring," Working Paper, MPRA.

Shavell, S., And L. Weiss (1979): "The Optimal Payment of Unemployment Insurance Benefits over Time," Journal of Political Economy, 87(6), 1347-1362.

Shimer, R., And I. Werning (2008): "Liquidity and Insurance for the Unemployed," American Economic Review, 98(5), 1922-42.

Wang, C., And S. Williamson (2002): "Moral Hazard, Optimal Unemployment Insurance, and Experience Rating," Journal of Monetary Economics, 49(7), 1337-1371.

Zhang, Y. (2009): "Dynamic Contracting with Persistent Shocks," Journal of Economic Theory, 144(2), 635-675. 


\section{Appendix A Data}

This section describes data on the accuracy of payments in the U.S. unemployment insurance system. We discuss three issues. First, we present an overall picture of unemployment insurance expenditures and briefly describe the system in place for determining the accuracy of the paid claims. Next, we provide details on the nature of "fraud" overpayments by cause, from 2005 - 2009. Finally, we present data on how these fraudulent payments were detected.

Total unemployment insurance expenditures in 2005, for example, were $\$ 30.2$ billion. These expenditures vary depending on aggregate economic activity; total expenditures during the recession in 2009 , for example, were $\$ 76.8$ billion. These benefits are paid out at the state level, with each state deciding its benefits and financing the benefits.

To determine the accuracy of these expenditures, the U.S. Department of Labor has a program, referred to as BAM (Benefit Accuracy Measurement). The BAM program chooses a random sample of weekly unemployment insurance claims and determines whether there were any overpayments. The investigators also interview some claimants if necessary.

The goal of the program is different from the goal of unemployment insurance fraud investigators. While the fraud investigators look to recapture overpayments, BAM investigators calculate statistics of the unemployment insurance program (see BAM State Operations Handbook ET No. 495, 4th Edition). Of the total overpayments determined by BAM, some represent simple errors in calculating benefits, while some represent fraud overpayments. Table A.1 details the various types of fraud overpayments from 2005-2009, averaged over all U.S. states.

Table A.1: Fraud Overpayments

\begin{tabular}{l|ccccc}
\hline & \multicolumn{4}{|c}{ Percent of Total Fraud } & Overpayments \\
\hline Cause & 2005 & 2006 & 2007 & 2008 & 2009 \\
\hline Concealed Earnings & 62.64 & 54.40 & 60.06 & 67.32 & 65.89 \\
Insufficient Job Search & 4.55 & 4.15 & 4.95 & 3.02 & 2.75 \\
Refused Suitable Offer & 0.63 & 0.36 & 0.80 & 0.36 & 0.77 \\
Quits & 12.78 & 16.41 & 7.06 & 5.04 & 5.14 \\
Fired & 4.27 & 4.60 & 13.29 & 12.69 & 9.61 \\
Unavailable for Work & 4.94 & 6.95 & 4.17 & 4.60 & 7.38 \\
Other & 10.20 & 13.14 & 9.67 & 6.97 & 8.46 \\
Total & 100.00 & 100.00 & 100.00 & 100.00 & 100.00 \\
\hline \hline
\end{tabular}

Source: Benefit Accuracy Measurement Program, U.S. Department of Labor

"Concealed Earnings" refers to payments to individuals who were simultaneously earning wages and collecting unemployment benefits. The category, "Insufficient Job Search," refers to cases where the individual did not meet the mandatory work search requirement (e.g., a minimum number of job applications must be filed each week). "Refused Suitable Offer" refers to cases where the individual was offered a job deemed suitable, but rejected it. "Quits" and "Fired" are separation issues, where Quits refers to payments to individuals who voluntarily left their job and Fired refers to payments to those who were fired 
from their job for a valid reason (e.g., poor performance or missing work). "Unavailable for Work" refers to payments to persons who cannot work (e.g., disability).

The UI system might incur another form of overpayment if workers strategically delay the start date of employment. That is, workers accept a job offer, but agree to start the job after their UI benets have expired. For example, Gauthier-Loiselle (2011) documents that the unemployment insurance expenditures are higher in Canada because of such cases. In the U.S. system, this is not considered fraud. Workers are entitled to collect UI benefits while waiting for an accepted job to start. Thus, the BAM data has no information on such cases, so they are not included in the fraud overpayments statistics.

The data in Table A.1 represent our calculations from the raw data files provided to us by the U.S. Department of Labor. More general statistics from the BAM program are available at http://www.ows.doleta.gov/unemploy/bqc.asp. Note, however, that our definitions and calculations differ slightly from those used in the BAM reports available online. In our calculations, we restrict attention to the cases of fraud that relate to the incentive problem we study in this paper and to the incentive problems that the existing literature has focused on.

Is Concealed Earnings fraud easy to detect? Table A.2 displays different detection technologies used by BAM. For example, "Verification of search contact" is when the BAM investigator verifies whether a potential job contact reported by the unemployed person is indeed true. "Claimant Interview" is an actual interview with the person collecting benefits, etc. The numbers in the table represent the percentage of each type of fraud uncovered by that particular method. More than 90 percent of the overpayments due to Concealed Earnings fraud were not detectable under the standard procedures available to the State authorities. This suggests that a more costly verification is necessary to uncover overpayments due to Concealed Earnings.

Table A.2: Detection Technologies, 2007

\begin{tabular}{l|c}
\hline Detection Method & $\begin{array}{c}\text { Percent of concealed earnings } \\
\text { fraud overpayments detected by method }\end{array}$ \\
\hline Verification of search contact & 1.31 \\
Verification of wages and/or separation & 62.02 \\
Claimant interview & 10.41 \\
Verification of eligibility with 3rd parties & 1.38 \\
Unemployment insurance records & 14.61 \\
Job/employment service records & 0.17 \\
Verification with union & 0.71 \\
Crossmatch with state directory of new hires & 7.52 \\
Crossmatch with state wage record files & 1.86 \\
\hline \hline
\end{tabular}

Source: Benefit Accuracy Measurement Program, U.S. Department of Labor

In Table 1 in Section 1, we reported that the overpayments due to Concealed Earnings fraud were almost twelve times the overpayments due to Insufficient Search fraud. Do the data understate the incidence of insufficient search? Recall that the BAM program measures only the extensive margin — whether the individual submits the required number 
of applications. It is possible that the unmeasured intensive margin — effort that turns an application into a job offer - is large enough to make the overpayments due to Insufficient Search comparable in magnitude to the overpayments due to Concealed Earnings. The following facts, however, suggest that the unmeasured component is unlikely to be large.

(i) Measured overpayments due to Insufficient Search have been declining: in 1988, they accounted for 34 percent of the total overpayments due to all fraud whereas in 2007 they accounted for less than 5 percent (the corresponding numbers for Concealed Earnings fraud were 41 percent and more than 60 percent).

(ii) The job search requirements that make an unemployed person eligible for benefits have increased over time, so the decline in the measured component is not due to changes in eligibility criteria. Hence, for the Insufficient Search overpayments to be the same in 2007 as those measured in 1988, the unmeasured component has to be almost six times that of the measured component in 2007.

(iii) If unmeasured efforts to translate a job application into a job offer were substantially higher in 2007, then the increase in efforts should manifest itself in a substantially higher transition rate from unemployment to employment. However, the evidence suggests that the transition rate is roughly constant: the quarterly rate was 0.31 for the period 1988-1997 and 0.33 for 1998-2007.

From a normative point of view, as noted in Section 1, the prevailing quantitative theory prescribes an intensive margin search effort that is less than the effort exerted under the current unemployment insurance program in the U.S. In other words, insufficient search is not a critical incentive problem in the U.S. (See also the evidence presented in Ashenfelter, Ashmore, and Deschenes (2005).)

\section{Appendix B Microfoundations for $E(t) \geq U(t)$}

Suppose that the worker can privately refuse a job offer. The timing in each period is as follows. The stochastic job opportunity arrives and the worker either receives an offer or does not. He then chooses to report the offer (if any) to the principal. Conditional on the report of an offer, the principal recommends the worker to either accept or reject the offer. The worker then chooses whether to follow the principal's recommendation. (In contrast, job acceptance is implicitly imposed in our model in Section 2.) Conditional on the report, the principal assigns current and future consumptions.

In such a job-refusal model, it is optimal for the principal to always recommend to the worker who reports an offer to accept the offer. Recommending "accept" minimizes the cost of delivering the promised utility since the worker's consumption is constant upon job acceptance and the principal gets the perpetual wage. Recommending "reject" means that the continuation contract involves additional uncertainty of job offers, reports, and incentive constraints. So the consumption cost of delivering the same promised utility is higher under "reject." Recall that, unlike Atkeson and Lucas (1995), we do not have disutility to working so it is optimal to always recommend "accept." 
The incentive compatibility for an agent with a job offer is as follows. If he reports his offer and receives a recommendation to accept, he strictly prefers "accept" to "reject." This is because rejecting the offer would not make him eligible for any unemployment insurance benefits, but would make him lose his wage income. If the agent does not report his offer, then either he rejects the offer and obtains $U(t)$, or he accepts the offer and commits fraud (i.e., he works and collects unemployment benefits at the same time). For the agent to truthfully report his offer, the utility of reporting and accepting the offer, $E(t)$, must be higher than both $U(t)$ and the utility he obtains by committing Concealed Earnings fraud. These Incentive Compatibility constraints are exactly conditions (2) and (3) in our model in Section 2.

\section{Appendix C Proofs}

Proof of Lemma 1: Suppose that a contract $\sigma \equiv\left\{\left(U(t), E(t), u(t), c^{U}(t), c^{E}(t), m_{i}\right) ; t \geq\right.$ $0, i \geq 1\}$ delivers the continuation utility $U$. Then, a contract

$$
\sigma_{\alpha} \equiv\left\{\left(\alpha U(t), \alpha E(t), \alpha u(t), c^{U}(t)-\log (\alpha) / \rho, c^{E}(t)-\log (\alpha) / \rho, m_{i}\right) ; t \geq 0, i \geq 1\right\}
$$

delivers $\alpha U$. The reverse is also true. Further, $\sigma$ is incentive compatible if and only if $\sigma_{\alpha}$ is incentive compatible. Therefore, $\left\{\left(U^{*}(t), E^{*}(t), u^{*}(t), c^{U *}(t), c^{E *}(t), m_{i}^{*}\right) ; t \geq 0, i \geq 1\right\}$ is the optimal contract to deliver $U$ if and only if

$$
\left\{\left(\alpha U^{*}(t), \alpha E^{*}(t), \alpha u^{*}(t), c^{U *}(t)-\log (\alpha) / \rho, c^{E *}(t)-\log (\alpha) / \rho, m_{i}^{*}\right) ; t \geq 0, i \geq 1\right\}
$$

is the optimal contract to deliver $\alpha U$.

LEMMA B.1 The promise-keeping constraint (1) and the incentive constraint (6) hold for all $0 \leq t<s \leq m_{1}$ if and only if

$$
\begin{aligned}
& U(s)-U(t)=\int_{t}^{s}((r+\pi) U(x)-\pi E(x)-r u(x)) d x, \\
& E(s)-E(t) \leq \int_{t}^{s}\left(r E(x)-r e^{-\rho w} u(x)\right) d x,
\end{aligned}
$$

hold for all $0 \leq t<s \leq m_{1}$. Taking the limit as s goes to $t$ yields the differential equations (10) and (11).

Proof. We only show the equivalence between (6) and (28), since the equivalence between (1) and (27) can be obtained similarly by replacing the inequalities below with equalities. 
Necessity: If (6) holds for all $t<s$, then

$$
\begin{aligned}
& E(t)+\int_{t}^{s}\left(r E(x)-r e^{-\rho w} u(x)\right) d x \\
\geq & \int_{t}^{s} e^{-r(x-t)} r e^{-\rho w} u(x) d x+e^{-r(s-t)} E(s) \\
& +\int_{t}^{s}\left(r\left(\int_{x}^{s} e^{-r(\eta-x)} r e^{-\rho w} u(\eta) d \eta+e^{-r(s-x)} E(s)\right)-r e^{-\rho w} u(x)\right) d x \\
= & \left(e^{-r(s-t)}+\int_{t}^{s} r e^{-r(s-x)} d x\right) E(s)+\int_{t}^{s}\left(e^{-r(x-t)}-1\right) r e^{-\rho w} u(x) d x \\
& +\int_{t}^{s} r\left(\int_{x}^{s} e^{-r(\eta-x)} r e^{-\rho w} u(\eta) d \eta\right) d x \\
= & E(s)+\int_{t}^{s}\left(e^{-r(x-t)}-1\right) r e^{-\rho w} u(x) d x+\int_{t}^{s}\left(\int_{t}^{\eta} r e^{-r(\eta-x)} d x\right) r e^{-\rho w} u(\eta) d \eta \\
= & E(s)+\int_{t}^{s}\left(e^{-r(x-t)}-1\right) r e^{-\rho w} u(x) d x+\int_{t}^{s}\left(1-e^{-r(\eta-t)}\right) r e^{-\rho w} u(\eta) d \eta \\
= & E(s) .
\end{aligned}
$$

Hence, inequality (28) is verified.

Sufficiency: Define an absolutely continuous function $f(\cdot)$ as

$$
f(s) \equiv \int_{t}^{s} e^{-r(x-t)} r e^{-\rho w} u(x) d x+e^{-r(s-t)}\left(E(t)+\int_{t}^{s}\left(r E(x)-r e^{-\rho w} u(x)\right) d x\right) .
$$

Because $f$ is absolutely continuous, it is differentiable almost everywhere (a.e.), and

$$
\begin{aligned}
f^{\prime}(s)= & e^{-r(s-t)} r e^{-\rho w} u(s)-r e^{-r(s-t)}\left(E(t)+\int_{t}^{s}\left(r E(x)-r e^{-\rho w} u(x)\right) d x\right) \\
& +e^{-r(s-t)}\left(r E(s)-r e^{-\rho w} u(s)\right) \\
= & r e^{-r(s-t)}\left(E(s)-E(t)-\int_{t}^{s}\left(r E(x)-r e^{-\rho w} u(x)\right) d x\right), \text { a.e. }
\end{aligned}
$$

If (28) holds, then $f^{\prime}(s) \leq 0$ a.e. Then, it follows from Theorem 29.15 in Aliprantis and Burkinshaw (1990) that

$$
f(s)=f(t)+\int_{t}^{s} f^{\prime}(x) d x \leq f(t)=E(t)
$$

Therefore,

$$
\int_{t}^{s} e^{-r(x-t)} r e^{-\rho w} u(x) d x+e^{-r(s-t)} E(s) \leq f(s) \leq E(t)
$$

which verifies inequality (6). 
Proof of Lemma 2: If (19), (20) and (21) all hold, we can substitute them into $(\Phi U+\lambda E)^{\prime}$ and obtain

$$
\begin{aligned}
(\Phi U+\lambda E)^{\prime} & =\Phi U^{\prime}+\lambda^{\prime} E+\lambda E^{\prime} \\
& =\Phi((r+\pi) U-\pi E-r u)+\pi\left(\Phi-c^{\prime}(E)+\lambda\right) E+\lambda\left(r E-r e^{-\rho w} u\right) \\
& =(r+\pi)(\Phi U+\lambda E)-\pi c^{\prime}(E) E-r\left(\Phi+e^{-\rho w} \lambda\right) u .
\end{aligned}
$$

Because $-c^{\prime}(E) E=\rho^{-1}$ and $-(\rho u)^{-1}=c^{\prime}(u)=\Phi+e^{-\rho w} \lambda$, we have

$$
(\Phi U+\lambda E)^{\prime}=(r+\pi)\left(\Phi U+\lambda E+\rho^{-1}\right) .
$$

Because $\Phi U(0)+\lambda(0) E(0)+\rho^{-1}=0$, it follows from (29) that $\Phi U(t)+\lambda(t) E(t)+\rho^{-1}=0$ for all $t \in\left[0, m_{1}\right]$.

On the other hand, if (20) and (21) hold and

$$
\Phi U(t)+\lambda(t) E(t)+\rho^{-1}=0, \quad \forall t \in\left[0, m_{1}\right],
$$

then $(\Phi U+\lambda E)^{\prime}=0$ for all $t \in\left[0, m_{1}\right]$. Then (19) can be derived by reversing the above steps.

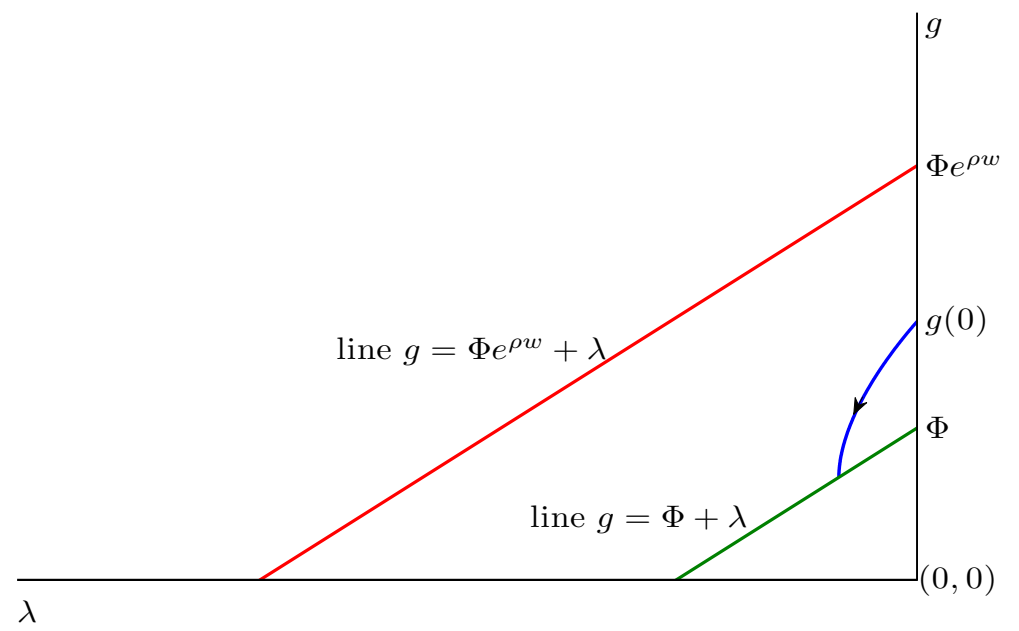

Figure 8: Phase Diagram for $(\lambda, g)$.

Proof of Lemma 3: First, it is convenient to transform the state variable $E$, which may approach $-\infty$, into a bounded one. To do so, we replace $E$ with

$$
g \equiv c^{\prime}(E)=-(\rho E)^{-1} .
$$

Now, the ODE system consists of (21) and

$$
g^{\prime}=\frac{E^{\prime}}{\rho E^{2}}=\frac{r g^{2}}{\Phi e^{\rho w}+\lambda}-r g,
$$


with boundary condition $g\left(m_{1}\right)=\Phi+\lambda\left(m_{1}\right)$ (Figure 8 shows the phase diagram). Let $m(g(0))$ be the time to hit the straight line $g=\Phi+\lambda$ starting with $(\lambda(0)=0, g(0))$.

Second, we show that $\lim _{g(0) \downarrow \Phi} m(g(0))=0$. If $\lambda=0$ and $g=\Phi$, then

$$
\begin{aligned}
(g-\lambda)^{\prime}(t) & =\left.\left(\frac{r g^{2}}{\Phi e^{\rho w}+\lambda}-r g+\pi(g-\lambda-\Phi)\right)\right|_{(\lambda, g)=(0, \Phi)} \\
& =\frac{r \Phi^{2}}{\Phi e^{\rho w}}-r \Phi<0 .
\end{aligned}
$$

Continuity of the ODE system (21), (30) implies that $(g-\lambda)^{\prime}(t)<0$ in a small neighborhood of $(0, \Phi)$. If $\lambda(0)=0$ and $g(0)$ approaches $\Phi$ from above, then $g(0)-\lambda(0)-\Phi$ approaches zero. Since the solution curve starting with $(0, g(0))$ will remain in the small neighborhood of $(0, \Phi)$ for a while, it will decrease and hit the line $g=\Phi+\lambda$ quickly if $g(0)-\lambda(0)-\Phi$ is sufficiently small.

Third, we show that $m(g(0))$ is strictly increasing in $g(0)$. Consider two paths that start with initial conditions $\left(0, g_{1}(0)\right)$ and $\left(0, g_{2}(0)\right)$, where $\Phi<g_{1}(0)<g_{2}(0)$. We will show that $g_{1}(t)-\lambda_{1}(t)<g_{2}(t)-\lambda_{2}(t)$ for all $t$. By contradiction, suppose $\left(g_{1}-\lambda_{1}\right)(t)=\left(g_{2}-\lambda_{2}\right)(t)$ for the first time at $t=t^{*}$. Because the two paths cannot cross, we cannot have that $g_{1}\left(t^{*}\right) \leq g_{2}\left(t^{*}\right)$. Then $g_{1}\left(t^{*}\right)>g_{2}\left(t^{*}\right)$ and $\lambda_{1}\left(t^{*}\right)>\lambda_{2}\left(t^{*}\right)$. Hence

$$
\begin{aligned}
\left(g_{1}-\lambda_{1}\right)^{\prime}\left(t^{*}\right) & =-\frac{r g_{1}}{\Phi e^{\rho w}+\lambda_{1}}\left(\Phi e^{\rho w}+\lambda_{1}-g_{1}\right)-\pi\left(\Phi+\lambda_{1}-g_{1}\right) \\
& <-\frac{r g_{2}}{\Phi e^{\rho w}+\lambda_{2}}\left(\Phi e^{\rho w}+\lambda_{2}-g_{2}\right)-\pi\left(\Phi+\lambda_{2}-g_{2}\right) \\
& =\left(g_{2}-\lambda_{2}\right)^{\prime}\left(t^{*}\right)
\end{aligned}
$$

where the inequality follows from $\frac{g_{1}}{\Phi e^{\rho w}+\lambda_{1}}>\frac{g_{2}}{\Phi e^{\rho w}+\lambda_{2}}$. That $\left(g_{1}-\lambda_{1}\right)^{\prime}\left(t^{*}\right)<\left(g_{2}-\lambda_{2}\right)^{\prime}\left(t^{*}\right)$ contradicts the facts that $\left(g_{1}-\lambda_{1}\right)\left(t^{*}\right)=\left(g_{2}-\lambda_{2}\right)\left(t^{*}\right)$ and $\left(g_{1}-\lambda_{1}\right)(t)<\left(g_{2}-\lambda_{2}\right)(t)$ for all $t<t^{*}$. Thus $g_{1}(t)-\lambda_{1}(t)<g_{2}(t)-\lambda_{2}(t)$ for all $t$, and the path $\left(\lambda_{1}(t), g_{1}(t)\right)$ reaches $g=\Phi+\lambda$ sooner.

Finally, we show there exists a unique $g(0)$ to satisfy $m(g(0))=m_{1}$ for any $m_{1}>0$. The second step in this proof shows that $\lim _{g(0) \downarrow \Phi} m(g(0))=0$. Part (ii) in Lemma B.2 (page 45) shows that $m(g(0))$ can be arbitrarily large with high values of $g(0)$. Hence, the existence of a unique solution to $m(g(0))=m_{1}$ follows from the intermediate value theorem and the monotonicity of $m(g(0))$ in $g(0)$.

Proof of Proposition 2: First, we show that $E, c^{U}, U$, and $\frac{U}{E}$ all fall on $\left[0, m_{1}\right]$. It follows from $g^{\prime}(t)<0$ that $E^{\prime}(t)=\rho E^{2}(t) g^{\prime}(t)<0$. Equation (13) implies that $u^{\prime}(t)=$ $\frac{e^{-\rho w} \lambda^{\prime}(t)}{c^{\prime \prime}(u)}<0$, or $\left(c^{U}\right)^{\prime}(t)<0$. Equation $(22)$ implies that $U^{\prime}(t)=-\Phi^{-1}(\lambda(t) E(t))^{\prime}<0$. Equation (22) also implies that $\frac{U}{E}=\Phi^{-1}(g-\lambda)$. Hence part (i) in Lemma B.2 implies that $\left(\frac{U}{E}\right)^{\prime}(t)<0$.

Second, to see the downward jump in $c^{U}(\cdot)$ at $m_{1}$, we show that

$$
\lim _{t \uparrow m_{1}} c^{\prime}(u(t))>\lim _{t \downarrow m_{1}} c^{\prime}(u(t)) .
$$


The left side is $\Phi+e^{-\rho w} \lambda\left(m_{1}\right)$ according to (13). To obtain the right side, we apply (13) to the interval $\left[m_{1}, 2 m_{1}\right)$, and obtain

$$
c^{\prime}(u(t))=C^{\prime}\left(U\left(m_{1}\right)\right)+e^{-\rho w} \tilde{\lambda}(t), \quad t \geq m_{1},
$$

where $\tilde{\lambda}$ denotes the multiplier $\lambda$ for the problem on the interval $\left[m_{1}, 2 m_{1}\right)$. Because $\tilde{\lambda}\left(m_{1}\right)=0$, we have $\lim _{t \downarrow m_{1}} c^{\prime}(u(t))=c^{\prime}\left(u\left(m_{1}\right)\right)=C^{\prime}\left(U\left(m_{1}\right)\right)+0=\Phi+\lambda\left(m_{1}\right)$. Therefore,

$$
\lim _{t \uparrow m_{1}} c^{\prime}(u(t))=\Phi+e^{-\rho w} \lambda\left(m_{1}\right)>\Phi+\lambda\left(m_{1}\right)=\lim _{t \downarrow m_{1}} c^{\prime}(u(t)) .
$$

Proof of Proposition 3: First, because $\frac{\Phi+e^{-\rho w} \lambda}{\Phi+\lambda}$ decreases in $\lambda$, and $\lambda\left(m_{1}\right)$ decreases in $g(0)$ and $m_{1}$, there is a unique value for $g(0)$ (as well as $m_{1}$ ) for a given $\psi$.

Second, to show that (23) is sufficient, we prove that

$$
\mathscr{C}^{\prime}\left(m_{1}\right)\left\{\begin{array}{cc}
<0, & m_{1}<m_{1}^{*} \\
>0, & m_{1}>m_{1}^{*}
\end{array}\right.
$$

This is because $\frac{\Phi+e^{-\rho w} \lambda\left(m_{1}\right)}{\Phi+\lambda\left(m_{1}\right)}$ strictly increases in $m_{1}: \frac{\Phi+e^{-\rho w} \lambda\left(m_{1}\right)}{\Phi+\lambda\left(m_{1}\right)}$ decreases in $\lambda\left(m_{1}\right)$ and the proof of Lemma 3 shows that $\lambda\left(m_{1}\right)$ decreases in $g(0)$ and $m_{1}$.

\section{Details in the computation of $\mathscr{C}^{\prime}\left(m_{1}\right)$}

Rewrite $\mathscr{C}\left(m_{1}\right)$ as

$$
\begin{aligned}
& \int_{0}^{m_{1}} e^{-(r+\pi) t}\left(\pi c\left(E^{m_{1}}\right)+r c\left(u^{m_{1}}\right)+\Phi\left((r+\pi) U^{m_{1}}-\pi E^{m_{1}}-r u^{m_{1}}-\left(U^{m_{1}}\right)^{\prime}\right)\right. \\
& \left.+\lambda^{m_{1}}\left(r E^{m_{1}}-r e^{-\rho w} u^{m_{1}}-\left(E^{m_{1}}\right)^{\prime}\right)\right) d t+e^{-(r+\pi) m_{1}}\left(\gamma+\psi+c\left(U^{m_{1}}\left(m_{1}\right)\right)\right) \\
& +e^{-(r+\pi) m_{1}} \lambda^{m_{1}}\left(m_{1}\right)\left(E^{m_{1}}\left(m_{1}\right)-U^{m_{1}}\left(m_{1}\right)\right),
\end{aligned}
$$

where we put a superscript $m_{1}$ on $U(\cdot), E(\cdot), u(\cdot)$, and $\lambda(\cdot)$ because these optimal paths rely on $m_{1}$. We use the Envelope theorem to simplify the computation of $\mathscr{C}^{\prime}\left(m_{1}\right)$. Since $U^{m_{1}}(t), E^{m_{1}}(t), u^{m_{1}}(t)$ are already optimally chosen at each $t$, we may view them as fixed when we vary $m_{1}$. Further, $U^{m_{1}}\left(m_{1}\right)$ and $E^{m_{1}}\left(m_{1}\right)$ can be viewed as varying only with the terminal date in the parenthesis. ${ }^{8}$ Viewed in this light, a small increment of $m_{1}$ is just an extrapolation of all time paths over a longer duration of unemployment, while the paths themselves are fixed. That is, we view all superscripts as being fixed and omit them when we calculate derivatives. Because $E\left(m_{1}\right)-U\left(m_{1}\right)=0$, we have

$$
\begin{aligned}
\mathscr{C}^{\prime}\left(m_{1}\right)= & e^{-(r+\pi) m_{1}}\left(\pi c\left(E\left(m_{1}\right)\right)+r c\left(u\left(m_{1}\right)\right)-(r+\pi)\left(\gamma+\psi+c\left(U\left(m_{1}\right)\right)\right)\right. \\
& \left.+c^{\prime}\left(U\left(m_{1}\right)\right) U^{\prime}\left(m_{1}\right)+\lambda\left(m_{1}\right)\left(E^{\prime}\left(m_{1}\right)-U^{\prime}\left(m_{1}\right)\right)\right) .
\end{aligned}
$$

\footnotetext{
${ }^{8}$ This is because $U^{\tilde{m}_{1}}\left(m_{1}\right)$ and $E^{\tilde{m}_{1}}\left(m_{1}\right)$ can be viewed as being fixed when we vary $\tilde{m}_{1}$.
} 
It follows from $c^{\prime}\left(U\left(m_{1}\right)\right)=\Phi+\lambda\left(m_{1}\right), \lambda^{\prime}\left(m_{1}\right)=0$ and Lemma 2 that

$$
\begin{aligned}
& c^{\prime}\left(U\left(m_{1}\right)\right) U^{\prime}\left(m_{1}\right)+\lambda\left(m_{1}\right)\left(E^{\prime}\left(m_{1}\right)-U^{\prime}\left(m_{1}\right)\right) \\
= & \Phi U^{\prime}\left(m_{1}\right)+\lambda\left(m_{1}\right) E^{\prime}\left(m_{1}\right)=\left(\Phi U\left(m_{1}\right)+\lambda\left(m_{1}\right) E\left(m_{1}\right)\right)^{\prime}=0 .
\end{aligned}
$$

Therefore,

$$
\begin{aligned}
\mathscr{C}^{\prime}\left(m_{1}\right) & =e^{-(r+\pi) m_{1}}\left(\pi c\left(E\left(m_{1}\right)\right)+r c\left(u\left(m_{1}\right)\right)-(r+\pi)\left(\gamma+\psi+c\left(U\left(m_{1}\right)\right)\right)\right) \\
& =e^{-(r+\pi) m_{1}}\left(r \rho^{-1} \log \left(\frac{\Phi+e^{-\rho w} \lambda\left(m_{1}\right)}{\Phi+\lambda\left(m_{1}\right)}\right)-(r+\pi)(\gamma+\psi)\right) .
\end{aligned}
$$

\section{Fixed-point condition for $\psi$}

The condition for $\psi$ is that $\psi$ is the fixed point of operator $T$, i.e.,

$$
\psi+c(U(0))=T(\psi)+c(U(0)) \equiv \min _{\sigma} C(\sigma) .
$$

We obtain $\psi$ from the first-order condition (23) for $m_{1}$,

$$
\psi=\frac{r \rho^{-1}}{r+\pi} \log \left(\frac{\Phi+e^{-\rho w} \lambda\left(m_{1}\right)}{\Phi+\lambda\left(m_{1}\right)}\right)-\gamma
$$

We obtain $T(\psi)$ from the HJB equation for the cost function at time zero

$$
\begin{aligned}
T(\psi)+c(U(0)) & =\frac{\pi c(E(0))+r c(u(0))+\Phi((r+\pi) U(0)-\pi E(0)-r u(0))}{r+\pi} \\
& =\frac{\pi}{r+\pi}\left(\frac{\Phi}{g(0)}-\log \left(\frac{\Phi}{g(0)}\right)-1\right)+c(U(0)) .
\end{aligned}
$$

The fixed-point condition $\psi=T(\psi)$ is rewritten as

$$
(r+\pi) \gamma=r \rho^{-1} \log \left(\frac{\Phi+e^{-\rho w} \lambda\left(m_{1}\right)}{\Phi+\lambda\left(m_{1}\right)}\right)-\pi\left(\frac{\Phi}{g(0)}-\log \left(\frac{\Phi}{g(0)}\right)-1\right) .
$$

Proposition 5 The path that satisfies (31) exists and is unique.

Proof. The existence of a path that satisfies (31) follows from the intermediate value theorem and the fact that right side of (31) is either extremely large or extremely small if we vary $g(0)$. To see this, note that the proof of Lemma 3 shows that $\lim _{g(0) \downarrow \Phi} m_{1}=0=$ $\lim _{g(0) \downarrow \Phi} \lambda\left(m_{1}\right)$. Therefore,

$$
\lim _{g(0) \downarrow \Phi} r \rho^{-1} \log \left(\frac{\Phi+e^{-\rho w} \lambda\left(m_{1}\right)}{\Phi+\lambda\left(m_{1}\right)}\right)-\pi\left(\frac{\Phi}{g(0)}-\log \left(\frac{\Phi}{g(0)}\right)-1\right)=0 .
$$

On the other hand, the proof of part (ii) of Lemma B.2 shows the existence of paths with $\lambda\left(m_{1}\right)$ approaching $-\Phi$ and $g(0) \in\left(\Phi, \Phi e^{\rho w}\right)$. For these paths, $\log \left(\frac{\Phi+e^{-\rho w} \lambda\left(m_{1}\right)}{\Phi+\lambda\left(m_{1}\right)}\right)$ can be arbitrarily large, while $\frac{\Phi}{g(0)}$ remains bounded. 
The uniqueness can be shown by contradiction. Suppose there are two paths satisfying (31). Associated with the two paths are two fixed points, $\psi<\tilde{\psi}$. Because the principal facing $\tilde{\psi}$ may monitor at $m_{1}(\psi)>0$ and adopt the optimal consumption paths under $\psi$,

$$
T(\tilde{\psi}) \leq \psi+e^{-(r+\pi) m_{1}(\psi)}(\tilde{\psi}-\psi)<\tilde{\psi}
$$

which contradicts the fact that $\tilde{\psi}$ is a fixed point.

LEMma B.2 Consider the ODE system (21), (30) with time running backwards, that is,

$$
\begin{aligned}
\lambda^{\prime} & =\pi(g-\Phi-\lambda), \\
g^{\prime} & =r g-\frac{r g^{2}}{\Phi e^{\rho w}+\lambda} .
\end{aligned}
$$

Suppose the initial condition is $(\lambda(0), g(0)=\Phi+\lambda(0)),-\Phi<\lambda(0)<0$, and $m^{-}(\lambda(0))$ denotes the first time to hit the $g$-axis, i.e., $m^{-}(\lambda(0))=\min _{t}\{t>0: \lambda(t)=0\}$.

(i) $(g-\lambda)^{\prime}(t)>0$ for all $t \in\left[0, m^{-}(\lambda(0))\right]$.

(ii) $m^{-}(\lambda(0))$ is finite, and $\lim _{\lambda(0) \downarrow-\Phi} m^{-}(\lambda(0))=\infty$.

ProOF.

(i) The path starting with $(\lambda(0), g(0)=\Phi+\lambda(0))$ has

$$
\begin{aligned}
\lambda^{\prime}(0) & =\pi(g(0)-\Phi-\lambda(0))=0, \\
g^{\prime}(0) & =r g(0)-\frac{r g(0)^{2}}{\Phi e^{\rho w}+\lambda(0)}>0 .
\end{aligned}
$$

Hence it moves beyond $g=\Phi+\lambda$ at time zero and satisfies $\Phi+\lambda<g<\Phi e^{\rho w}+\lambda$ before reaching the $g$-axis. If $\Phi+\lambda<g<\Phi e^{\rho w}+\lambda$, then $g^{\prime}>0$ and $\lambda^{\prime}>0$.

To show that $(g-\lambda)^{\prime}(t)>0$ for all $t \in\left[0, m^{-}(\lambda(0))\right]$, suppose to the contrary that $(g-\lambda)^{\prime}(s) \leq 0$ for some $s$. Let $t^{*}=\min _{s}\left\{s>0:(g-\lambda)^{\prime}(s) \leq 0\right\}$. It is easily seen that $(g-\lambda)^{\prime}\left(t^{*}\right)=0$ and $(g-\lambda)^{\prime \prime}\left(t^{*}\right) \leq 0$. Since $(g-\lambda)^{\prime}=r g-\frac{r g^{2}}{\Phi e^{\rho w}+\lambda}-\pi(g-\Phi-\lambda)$,

$$
\begin{aligned}
(g-\lambda)^{\prime \prime}\left(t^{*}\right) & =\left(r-\frac{2 r g\left(\Phi e^{\rho w}+\lambda\right)}{\left(\Phi e^{\rho w}+\lambda\right)^{2}}-\pi\right) g^{\prime}\left(t^{*}\right)+\left(\frac{r g^{2}}{\left(\Phi e^{\rho w}+\lambda\right)^{2}}+\pi\right) \lambda^{\prime}\left(t^{*}\right) \\
& =\left(r+\frac{r g^{2}-2 r g\left(\Phi e^{\rho w}+\lambda\right)}{\left(\Phi e^{\rho w}+\lambda\right)^{2}}\right) g^{\prime}\left(t^{*}\right) \\
& =r \frac{\left(\Phi e^{\rho w}+\lambda-g\right)^{2}}{\left(\Phi e^{\rho w}+\lambda\right)^{2}} g^{\prime}\left(t^{*}\right)>0,
\end{aligned}
$$

where the second equality follows from $g^{\prime}\left(t^{*}\right)=\lambda^{\prime}\left(t^{*}\right)$. This contradicts that $(g-$ $\lambda)^{\prime \prime}\left(t^{*}\right) \leq 0$. 
(ii) First, we show that $m^{-}(\lambda(0))$ is finite. We know from part (i) that $\lambda^{\prime}>0$. It follows from $(32)$ and $(g-\lambda)^{\prime}>0$ in part (i) that

$$
\lambda^{\prime \prime}=\pi(g-\lambda)^{\prime}>0
$$

Hence starting from $\lambda(0)<0, \lambda(t)$ accelerates and will reach zero in finite time.

Second, we show that $\lim _{\lambda(0) \downarrow-\Phi} m^{-}(\lambda(0))=\infty$. If $\lambda(0)=-\Phi$ and $g(0)=0$, then

$$
\begin{aligned}
& \lambda^{\prime}(0)=\pi(g(0)-\Phi-\lambda(0))=0, \\
& g^{\prime}(0)=r g(0)-\frac{r g(0)^{2}}{\Phi e^{\rho w}+\lambda(0)}=0 .
\end{aligned}
$$

Continuity of the ODE system (32), (33) implies that $(\lambda, g)$ will stay in a small neighborhood of $(-\Phi, 0)$ for a long duration if $\lambda(0)$ is sufficiently close to $-\Phi$ and $g(0)=\Phi+\lambda(0)$. Therefore, $\lim _{\lambda(0) \downarrow-\Phi} m^{-}(\lambda(0))=\infty$.

\section{Appendix D Stochastic Verification}

\section{D.1 Construction of a Contract}

To prove Proposition 4, we first construct a contract $\sigma^{*}$ in which $E(t)>U(t)$ implies $p(t)=0$, and $E(t)=U(t)$ implies $p(t)>0$. This contract has the features described in Proposition 4 , and in the next section we verify it is indeed optimal.

First, since the principal does not monitor in this contract when $E>U$, we still use the ODE system (20), (21) to find a solution path in the interval $[0, N]$, where $N$ satisfies

$$
-\int_{0}^{N} \lambda(t)\left(r E-r e^{-\rho w} u\right) d t-\lambda(N)\left(e^{\rho \phi}-1\right) E(N)+\gamma=0 .
$$

The two boundary conditions for the ODE system (20), (21) are still $\lambda(0)=0$ and $E(N)=$ $-\rho^{-1}(\Phi+\lambda(N))^{-1}$.

LEMMA 4 The $N$ that satisfies (34) exists and is unique.

Proof. For uniqueness, we show that $f(N) \equiv-\int_{0}^{N} \lambda(t)\left(r E-r e^{-\rho w} u\right) d t-\lambda(N)\left(e^{\phi}-\right.$ 1) $E(N)$ decreases with $N$. Since both $\lambda(N)$ and $E(N)$ are negative and decreasing with $N,-\lambda(N)\left(e^{\phi}-1\right) E(N)$ decreases with $N$. Moreover,

$$
-\lambda\left(r E-r e^{-\rho w} u\right)=\frac{r|\lambda|}{g\left(\Phi e^{\rho w}+\lambda\right)}\left(g-\lambda-\Phi e^{\rho w}\right) .
$$

For fixed $t, \frac{r|\lambda|}{g\left(\Phi e^{\rho w}+\lambda\right)}$ increases with $N$, while $\left(g-\lambda-\Phi e^{\rho w}\right)$ is more negative with higher $N$. Therefore, $-\int_{0}^{N} \lambda\left(r E-r e^{-\rho w} u\right) d t$ decreases with $N$ too. For existence, note that 
$\lim _{N \rightarrow 0} f(N)=0$. Because $\lim _{N \rightarrow \infty} \lambda(N)=-\Phi$ and $\lim _{N \rightarrow \infty} E(N)=-\infty$, we have $\lim _{N \rightarrow \infty} f(N)=-\infty$.

Second, choose $p>0$ after $N$ so that the state vector stays on the 45-degree line before the monitoring arrives, i.e., $U(t)=E(t)$ for all $t \geq N$. Choosing $\tilde{U}(N)=U(0)=-\frac{1}{\rho \Phi}$ and solving the equation $U^{\prime}(N)=E^{\prime}(N)$, we have

$$
p=\frac{r\left(1-e^{-\rho w}\right)\left(\Phi+e^{-\rho w} \lambda(N)\right)^{-1}}{e^{\rho \phi}(\Phi+\lambda(N))^{-1}-\Phi^{-1}}>0 .
$$

Note that $p$ is independent of $\Phi$. This also implies that $p>0$ is time invariant after $N$ because $U(t)=E(t)$ for $t \geq N$.

Third, the constructed solution path defines a contract $\sigma^{*}$ as follows. For each $t \in[0, N]$, the policy $u(t)$ is obtained by the first-order condition (13)

$$
u(t)=-\frac{1}{\rho\left(\Phi+e^{-\rho w} \lambda(t)\right)} .
$$

If $t \geq N$, then the state vector moves along the 45-degree line, and $u(t)$ is always proportional to $(U(t), E(t))$. That is, for all $t \geq N$,

$$
\frac{u^{\prime}(t)}{u(t)}=\frac{E^{\prime}(t)}{E(t)}=\frac{U^{\prime}(t)}{U(t)}=r-\frac{r(\Phi+\lambda(N))}{\Phi+e^{-\rho w} \lambda(N)}+p\left(1-\frac{\Phi+\lambda(N)}{\Phi}\right)>0 .
$$

The contract $\sigma^{*}$ is defined by (34-37), and the property that the continuation contract after a monitoring at $t \geq N$ starts a new cycle, in which the continuation utility is

$\tilde{U}(t)=\frac{\Phi+\lambda(N)}{\Phi} U(t)$ instead of $U(0)$. In this construction, $\sigma^{*}$ has the features mentioned in Proposition 4.

\section{D.2 Optimality of the Contract}

First, using the path obtained in Lemma 4, we construct a cost function $C$ as

$$
\begin{aligned}
(r+\pi) C(U(t), E(t))= & \pi c(E(t))+r c(u(t))+\Phi((r+\pi) U(t)-\pi E(t)-r u(t)) \\
& +\lambda(t)\left(r E(t)-r e^{-\rho w} u(t)\right) .
\end{aligned}
$$

Lemma $5 C_{U}(U(t), E(t))=\Phi$, and $C_{E}(U(t), E(t))=\lambda(t)$.

Proof. Differentiate (38) with respect to $t$, we have

$(r+\pi)\left(C_{U} U^{\prime}(t)+C_{E} E^{\prime}(t)\right)=\pi c^{\prime}(E) E^{\prime}(t)+\Phi\left((r+\pi) U^{\prime}(t)-\pi E^{\prime}(t)\right)+\lambda(t) r E^{\prime}(t)+\lambda^{\prime}(t) E^{\prime}(t)$,

which, after substituting $\lambda^{\prime}(t)=\pi\left(\Phi-c^{\prime}(E)+\lambda\right)$, becomes

$$
C_{U} U^{\prime}(t)+C_{E} E^{\prime}(t)=\Phi U^{\prime}(t)+\lambda(t) E^{\prime}(t) .
$$

Homogeneity of $C(\cdot, \cdot)$ implies that $C_{U} U(t)+C_{E} E(t)+\rho^{-1}=0=\Phi U(t)+\lambda(t) E(t)+\rho^{-1}$. Because the vectors $\left(U^{\prime}(t), E^{\prime}(t)\right)$ and $(U(t), E(t))$ are linearly independent (we have shown 
that $\left(\frac{U}{E}\right)^{\prime}(t)<0$ in the proof of Proposition 2, which is $\left.\frac{E^{\prime}(t)}{E(t)}>\frac{U^{\prime}(t)}{U(t)}\right)$, we have $C_{U}=\Phi$ and $C_{E}=\lambda(t)$.

Second, we verify that the cost function $C$ satisfies the HJB equation:

$$
\begin{aligned}
(r+\pi) C(U, E)=\min _{u, p, \tilde{U}, \tilde{E}} & \{r c(u)+\pi c(E)+p(C(\tilde{U}, \tilde{E})+\gamma-C(U, E)) \\
& +C_{U}(r(U-u)-\pi(E-U)-p(\tilde{U}-U)) \\
& \left.+C_{E}\left(r E-r e^{-\rho w} u-p\left(e^{\rho \phi}-1\right) E\right)\right\},
\end{aligned}
$$

where $(\tilde{U}, \tilde{E})$ is the new state vector the principal chooses after the next monitoring.

Lemma 6 The $C(\cdot, \cdot)$ defined in (38) satisfies (39).

Proof. The only differences between (38) and (39) are the terms associated with arrival rate $p$, which will be shown to be zero in this proof. Fix a $t \in[0, N]$ and consider the HJB equation at $(U(t), E(t))$. The first-order condition for $\tilde{U}$ implies that $\tilde{U}=U(0)$. Then we have

$$
\begin{gathered}
C(\tilde{U}, \tilde{E})+\gamma-C(U, E)-\Phi(\tilde{U}-U)-C_{E}\left(e^{\rho \phi}-1\right) E \\
=-\int_{0}^{t} \lambda(s)\left(r E(s)-r e^{-\rho w} u(s)\right) d s-\lambda(t)\left(e^{\phi}-1\right) E(t)+\gamma .
\end{gathered}
$$

The above is decreasing in $t$ because $\lambda(t)<0$, and $E(t)<0$ both decrease in $t$. Moreover, the integral $-\int_{0}^{t} \lambda(s)\left(r E(s)-r e^{-\rho w} u(s)\right) d s$ decreases in $t$ because

$$
r E(t)-r e^{-\rho w} u(t)=E^{\prime}(t)=\rho E^{2}(t) g^{\prime}(t)<0 .
$$

Therefore, the definition of $N$ in (34) implies that

$$
C(\tilde{U}, \tilde{E})+\gamma-C(U, E)-\Phi(\tilde{U}-U)-C_{E}\left(e^{\rho \phi}-1\right) E \begin{cases}>0, & \text { if } t<N \\ =0, & \text { if } t=N\end{cases}
$$

This implies that

$$
\min _{p \geq 0} p\left(C(\tilde{U}, \tilde{E})+\gamma-C(U, E)-\Phi(\tilde{U}-U)-C_{E}\left(e^{\rho \phi}-1\right) E\right)=0
$$

which finishes the proof.

Finally, to complete the proof of Proposition 4, we show that the contract $\sigma^{*}$ is optimal.

Proof of Proposition 4: Because the technique of using the HJB equation to verify optimality is standard, we spare the reader of detailed steps. Given the initial promised utilities $(U, E)$, we need to verify that

(i) The cost of the contract $\sigma^{*}$ is $C(U, E)$. 
(ii) The costs of other I.C. contracts are weakly higher than $C(U, E)$.

We only verify (ii) here, since the proof for (i) can be obtained simply by replacing the following inequalities with equalities.

To see that the cost of an I.C. contract $\left\{\left(\tilde{c}^{E}(t), \tilde{c}^{U}(t), \tilde{p}(t)\right) ; t \geq 0\right\}$ is higher than $C(U, E)$, define

$$
\begin{aligned}
h(T)= & \int_{0}^{T} e^{-(r+\pi) t-\int_{0}^{t} \tilde{p}(x) d x}\left(\pi c(\tilde{E}(t))+r \tilde{c}^{U}(t)+\tilde{p}(t)(C(\tilde{U}(t), \tilde{E}(t))+\gamma)\right) d t \\
& +e^{-(r+\pi) T-\int_{0}^{T} \tilde{p}(x) d x} C(U(T), E(T)) .
\end{aligned}
$$

The HJB equation implies that $f^{\prime}(T) \geq 0$. Therefore, $h(T)$ increases in $T$, and

$$
C(U, E)=h(0) \leq h(T)
$$

Taking limit $T \rightarrow \infty$, we have

$$
C(U, E) \leq \int_{0}^{\infty} e^{-(r+\pi) t-\int_{0}^{t} \tilde{p}(x) d x}\left(\pi c(\tilde{E}(t))+r \tilde{c}^{U}(t)+\tilde{p}(t)(C(\tilde{U}(t), \tilde{E}(t))+\gamma)\right) d t
$$

which can be rewritten as

$$
\begin{aligned}
C(U, E) \leq & E\left[\int_{0}^{\tau_{1}} e^{-r t}\left(\pi c(\tilde{E}(t))+r \tilde{c}^{U}(t)\right) d t\right]+E\left[e^{-r \tau_{1}} \gamma\right] \\
& +E\left[e^{-r \tau_{1}} C\left(\tilde{U}\left(\tau_{1}\right), \tilde{E}\left(\tau_{1}\right)\right)\right]
\end{aligned}
$$

where $\tau_{1}$ is the first monitoring time and $\left(\tilde{U}\left(\tau_{1}\right), \tilde{E}\left(\tau_{1}\right)\right)$ is the state vector immediately after monitoring. Inductively, we obtain

$$
\begin{aligned}
C(U, E) \leq & E\left[\int_{0}^{\tau_{n}} e^{-r t}\left(\pi c(\tilde{E}(t))+r \tilde{c}^{U}(t)\right) d t\right]+E\left[\sum_{i=1}^{n} e^{-r \tau_{i}} \gamma\right] \\
& +E\left[e^{-r \tau_{n}} C\left(\tilde{U}\left(\tau_{n}\right), \tilde{E}\left(\tau_{n}\right)\right)\right]
\end{aligned}
$$

where $\tau_{n}$ is the $n$th monitoring time. Without loss of generality, we may assume that $\lim _{n \rightarrow \infty} \tau_{n}=\infty$ almost surely (otherwise the principal monitors infinitely many times in finite time and the monitoring cost is infinity). Taking limit $n \rightarrow \infty$ yields

$$
C(U, E) \leq E\left[\int_{0}^{\infty} e^{-r t}\left(\pi c(\tilde{E}(t))+r \tilde{c}^{U}(t)\right) d t\right]+E\left[\sum_{i=1}^{\infty} e^{-r \tau_{i}} \gamma\right]
$$




\section{Appendix E Imperfect Detection}

This section presents a version of the stochastic verification model where detection is imperfect. Specifically, there is a positive probability $\varpi>0$ of monitoring error. In the event of monitoring error, an unemployed worker is labeled as employed. If an unemployed worker is monitored after reporting unemployment, the principal observes either an unemployed signal $\mathcal{U}$ with probability $1-\varpi$ or an employed signal $\mathcal{E}$ with probability $\varpi$. On the other hand, there is no monitoring error that labels an employed worker as being unemployed, i.e., if an employed worker is monitored after reporting unemployment, the principal observes $\mathcal{E}$ with probability one.

The timing of the problem is similar to the stochastic verification case in Section 7 . The planner still chooses the arrival rate of monitoring, $p(t)$, conditional on the report of unemployment in period $t$. There are, however, two differences in the case of imperfect detection. First, the planner assigns continuation utilities based not only on whether or not monitoring occurs (as above) but also on the signal from monitoring. Let $U_{\mathcal{U}}(t)$ and $U_{\mathcal{E}}(t)$ be the continuation utilities of a monitored unemployed worker with signals $\mathcal{U}$ and $\mathcal{E}$ at $t$, respectively. Let $E_{\mathcal{E}}(t)$ be the continuation utility of a monitored employed worker (whose signal can only be $\mathcal{E}$ ) at $t$. Finally, $E_{\mathcal{U}}(t)$ is the continuation utility of a monitored unemployed worker with signal $\mathcal{U}$ who transited to employment immediately after being monitored. Second, the penalty is exogenous in the case of perfect detection above, but is endogenous with imperfect detection.

Similar to (24) and (25), the promise-keeping constraint and incentive constraint are

$$
\begin{aligned}
U^{\prime} & =r(U-u)-\pi(E-U)-p\left[(1-\varpi) U_{\mathcal{U}}+\varpi U_{\mathcal{E}}-U\right] \\
E^{\prime} & \leq r E-r e^{-\rho w} u-p\left(E_{\mathcal{E}}-E\right) .
\end{aligned}
$$

There are two differences between these two equations and (24) and (25). First, the promisekeeping constraint (40) incorporates the possibility that an unemployed worker may be labeled as employed after monitoring. Second, in (25) the last term on the right-hand side results from the exogenous and finite penalty, $\phi$, whereas in (41) the last term allows the penalty $E_{\mathcal{E}}$ to be endogenous.

The main results from the perfection detection case and stochastic monitoring still hold here. That is, the optimal monitoring mechanism consists of cycles. Within each cycle, there exists some $N$ such that the planner sets $p=0$ before $N$, and then monitors at rate $p$ thereafter. Formally we state the following proposition.

Proposition 6 There exists an $N>0$ such that the principal monitors the unemployed with a constant arrival rate $p>0$ if and only if $t \geq N$. Before $N$, the time path $(U(\cdot), E(\cdot)$ ) converges to the 45-degree line; after $N$, the utility pair $(U(t), E(t))$ remains stationary (i.e., $U(t)=E(t)=U(N)=E(N)$ for all $t \geq N$ ) until the worker is randomly drawn to be monitored. If the observed signal from monitoring is $\mathcal{E}$, the worker is punished, $U_{\mathcal{E}}=E_{\mathcal{E}}<U(N)$. If the signal is $\mathcal{U}$, the worker is rewarded, $U_{\mathcal{U}}>U(N)$, and the contract enters a new cycle. 